\title{
Neutral Gas Outflows and Inflows in Infrared-Faint Seyfert Galaxies
}

\author{
Hannah B. Krug ${ }^{1}$, David S. N. Rupke ${ }^{2}$ \& Sylvain Veilleux ${ }^{1,3}$
}

\begin{abstract}
Previous studies of the Na I D interstellar absorption line doublet have shown that galactic winds occur in most galaxies with high infrared luminosities. However, in infrared-bright composite systems where a starburst coexists with an active galactic nucleus (AGN), it is unclear whether the starburst, the AGN, or both are driving the outflows. The present paper describes the results from a search for outflows in 35 infrared-faint Seyferts with $10^{9.9}<L_{\mathrm{IR}} / L_{\odot}<10^{11}$, or, equivalently, star formation rates (SFR) of $\sim 0.4-9 \mathrm{M}_{\odot} \mathrm{yr}^{-1}$, to attempt to isolate the source of the outflow. We find that the outflow detection rates for the infrared-faint Seyfert 1s (6\%) and Seyfert 2s (18\%) are lower than previously reported for infrared-luminous Seyfert 1s (50\%) and Seyfert 2s (45\%). The outflow kinematics of infrared-faint and infrared-bright Seyfert 2 galaxies resemble those of starburst galaxies, while the outflow velocities in Seyfert 1 galaxies are significantly larger. Taken together, these results suggest that the AGN does not play a significant role in driving the outflows in most infrared-faint and infrared-bright systems, except the high-velocity outflows seen in Seyfert 1 galaxies. Another striking result of this study is the high rate of detection of inflows in infraredfaint galaxies (39\% of Seyfert 1s, 35\% of Seyfert 2s), significantly larger than in infrared-luminous Seyferts (15\%). This inflow may be contributing to the feeding of the AGN in these galaxies, and potentially provides more than enough material to power the observed nuclear activity over typical AGN lifetimes.
\end{abstract}

Subject headings: galaxies: active — galaxies: Seyfert — galaxies: quasars: absorption lines — line: profiles — ISM: jets and outflows - ISM: kinematics and dynamics

\footnotetext{
${ }^{1}$ Department of Astronomy, University of Maryland, College Park, MD 20742; E-mail: hkrug@astro.umd.edu, veilleux@astro.umd.edu

${ }^{2}$ Institute for Astronomy, University of Hawaii, 2680 Woodlawn Drive, Honolulu, HI 96822; E-mail: drupke@ifa.hawaii.edu

${ }^{3}$ Also: Max-Planck-Institut für extraterrestrische Physik, Postfach 1312, D-85741 Garching, Germany
} 


\section{INTRODUCTION}

Galactic-scale gas outflows appear to play an important role in the evolution of the universe, possibly providing an explanation for a number of cosmological questions (Veilleux et al. 2005 and references therein). These galactic outflows are frequently observed in local galaxies with high star formation rates and/or an active nucleus, often extending on a scale of order of a few kiloparsecs or larger.

The high frequency of outflows measured in high-redshift, actively star-forming objects such as Lyman break galaxies suggests that outflows are a common stage in galactic evolution, with wind velocities decreasing over time (Shapley et al. 2003; Ferrara \& Ricotti 2006). Such outflows may be responsible for the deficit of baryons seen in our own Milky Way galaxy and the mass-metallicity relation in external galaxies, if winds are capable of preferentially ejecting metals into the intergalactic medium (Larson 1974; Garnett 2002; Tremonti et al. 2004). The intergalactic medium can be heated as well as enriched with metals by these galactic outflows, since energy inputs into the IGM on the order of $10^{56}$ ergs have been measured from galaxies with strong outflows (Croston et al. 2008). Additionally, outflows may quench star formation by heating up cold gas and ejecting it from the host (Binney 2004; Scannapieco et al. 2005). Specifically, AGN-powered outflows have been proposed as the cause of the drop in AGN luminosity at low redshift and the cutoff at high luminosity of the galaxy luminosity function (Somerville \& Primack 1999; Cole et al. 2000; Thacker et al. 2006). These winds may also limit black hole and spheroid growth, and be responsible for the observed tight correlation between black hole mass and galactic spheroid mass (Ferrarese \& Merritt 2000; McLure \& Dunlop 2002; Granato et al. 2004). Finally, winds driven by AGN and/or starbursts may help remove enough nuclear material with low angular momentum early on in the evolution of gas-rich systems to aid in the formation of large disks with high specific angular momentum, more in line with the observations (Binney et al. 2001; Kormendy \& Kennicutt 2004).

Studies performed on actively star-forming galaxies, near and far, have revealed that outflow energetics increase with infrared $(8-1000 \mu \mathrm{m})$ luminosity, $L_{\mathrm{IR}}$, or equivalently, star formation rate (Martin 2005; Rupke et al. 2005a, hereafter RVS05a; Rupke et al. 2005b, hereafter RVS05b; Sato et al. 2009; Weiner et al. 2009). Outflows have also been detected in composite galaxies with both starbursts and AGN (e.g., Irwin \& Saikia 2003; Hota \& Saikia 2006). Seyfert 1 composites show significantly higher outflow velocities than pure starbursts and Seyfert 2 composites (Rupke et al. 2005c, hereafter RVS05c). Hydrodynamical simulations of starburst-driven outflows (e.g., Thacker et al. 2006; Cooper et al. 2008) have difficulties reproducing the very high velocities detected in Seyfert 1 composites. The AGN in these objects thus appear to play a significant role in driving these outflows, at least on 
small scales (Crenshaw et al. 2003b). The situation in Seyfert 2 composites is more ambiguous - the AGN or starburst or both could be powering the outflow in these systems (Schiano 1985).

The focus of this paper is on "pure" Seyfert galaxies with weak infrared starbursts. Our sample consists of 35 galaxies that are faint in the infrared $\left(10^{9.9}<L_{\mathrm{IR}} / L_{\odot}<10^{11.2}\right)$, equally split between Seyfert 1s and Seyfert 2s. We compare the results from our study with those from previous studies of starburst and Seyfert ultraluminous infrared galaxies (ULIRGs; $L_{\mathrm{IR}} / L_{\odot} \geq 10^{12}$ ) and luminous infrared galaxies (LIRGs; $10^{11}<L_{\mathrm{IR}} / L_{\odot}<10^{12}$ ) to isolate the role of the AGN in powering these outflows. As in previous studies, the IR-faint objects were observed in the Na I D $\lambda \lambda 5890,5896$ doublet absorption feature. This Na feature has a low ionization potential $(5.1 \mathrm{eV})$, so it probes neutral gas, and it can be used to study the ISM due to its high interstellar abundance. All objects in the sample have $z<0.05$, and thus the Na I D feature is found in the optical. Velocity components in Na I D absorption are unambiguous indicators of outflowing (blueshifted velocities) or inflowing (redshifted velocities) gas, as there must be a continuum source behind the absorber.

The organization of this paper is as follows. The sample is described in Section 2 , including methods of selection and sample properties in terms of redshifts, star formation rates, and spectral types. The observations are discussed in Section 3. Section 4 describes the line fitting, the derivations of the velocities and column densities, and estimates of the expected stellar contributions to the measured Na I D absorption. In Section 5 we present the results on outflows, starting with the Seyfert $2 \mathrm{~s}$ and followed by the Seyfert 1s. Next, Section 6 describes the results on inflows in the same fashion as Section 5. This is followed by a discussion of the implications of our findings in Section 7, including comparisons with previous studies, dynamical estimates, and possible connections between inflows and nuclear structures. We conclude in Section 8 with a summary of our findings.

All calculations in this study assume present-day cosmological parameter values, with $H_{0}=75 \mathrm{~km} \mathrm{~s}^{-1} \mathrm{Mpc}^{-1}, \Omega_{m}=0.3$, and $\Omega_{\Lambda}=0.7$. All wavelengths quoted are vacuum wavelengths.

\section{SAMPLE}

The objects in the sample were selected using three main criteria: They had to be optically classified as Seyferts and had to be faint in the infrared $\left(L_{\mathrm{IR}} \lesssim 10^{11} L_{\odot}\right)$. All objects in the sample were also selected to have $z<0.05$ to focus on the brightest sources and obtain reliable measurements. 
Most galaxies in the sample are well-studied Seyfert galaxies with extensive ancillary data (e.g. Whittle 1992a; Nelson \& Whittle 1995). Roughly equal numbers of Seyfert 1 and Seyfert 2 galaxies were chosen to allow meaningful comparisons with the study of RVS05c on ULIRG Seyferts. The bolometric luminosities of the AGN in the present sample are well matched to those in the composite systems of RVS05c. This allows us to make direct comparisons between the two samples. Basic properties for the galaxies in our sample can be found in Table 1. The last column in that table lists the references used for the compilation.

\subsection{Redshifts}

Not all objects in this survey have well-constrained published redshift measurements, so preference was given to values from stellar absorption lines (Nelson \& Whittle 1995; Wegner et al. 2003) when possible, followed by redshifts taken from HI lines (Nelson \& Whittle 1995; Springob et al. 2005). Redshifts measured from data in the present survey were used if neither of the preceding were available, but were given low priority due to systematic errors $\left(\sim 10^{-4}\right)$ associated with these measurements (e.g. it is possible that part of the narrow line region (NLR) is outflowing so the velocities based on the narrow emission lines are not necessarily representative of the systemic velocity). In this case, IRAF was used to measure central wavelength of various emission lines, including [O I] $\lambda \lambda 6300,6364$, [N II] $\lambda \lambda 6548,6583$, and [S II] $\lambda \lambda 6716,6731$, and redshifts were calculated from those values.

\subsection{Star Formation Rates}

To estimate the star formation rates in these objects, we use their far-infrared luminosity, $L(40-120 \mu \mathrm{m})$, under the assumption that the AGN does not contribute significantly to $L_{\text {FIR }}$ (Schweitzer et al. 2006). This is probably a good first-order approximation although AGN may contribute $\sim 10-20 \%$ to $L_{\mathrm{FIR}}$ (Netzer et al. 2007). $L_{\mathrm{FIR}}$ is calculated using the prescription of Sanders \& Mirabel (1996), using data from the IRAS Faint Source and IRAS Point Source Catalogs. $L_{\mathrm{FIR}}$ is then used to calculate SFR using the relation in Kennicutt (1998), $S F R=L_{\mathrm{FIR}} /\left(5.8 \times 10^{9} L_{\odot}\right) M_{\odot} \mathrm{yr}^{-1}$. The far-infrared luminosities and star formation rates of the objects in our sample are listed in Table 1 . 


\subsection{Spectral Types}

Spectral classifications were taken from the NASA Extragalactic Database 1 and confirmed through examination of emission lines in the spectra and references in the literature. Seyfert $1-1.5$ were labeled Seyfert 1s for the purpose of this study, while Seyfert 1.8 - 2 were labeled Seyfert 2s. This is in keeping with the classification scheme of RVS05c and allows for comparisons of our results on Seyfert 1 and Seyfert 2 galaxies with those of previous studies.

\section{OBSERVATIONS}

All observations were taken over the course of three different observing runs on the Kitt Peak 4-meter telescope. Observing runs, exposure times, and slit position angles for all objects are listed in Table 1. All data were taken using the Ritchey-Chrétien Spectrograph with a moderate-resolution grating, KPC-18C, in the first order along with the GG-475 blocking filter. The wavelength range was $1700 \AA$, allowing measurements of both the Na I D absorption doublet and $\mathrm{H} \alpha+[\mathrm{N} \mathrm{II}]$ emission complex in the same exposure. This combination provides an average resolution of $85 \mathrm{~km} \mathrm{~s}^{-1}$ with a $1^{\prime \prime} .25$ slit. Median signal-to-noise ratio near Na I D was 85 per $\AA$ and ranged from 23 to 337 per $\AA$, with seeing ranging from $\sim 1$ to $2^{\prime \prime}$ on average.

\section{ANALYSIS}

\subsection{Line Fitting}

Extraction and fitting procedures were performed as described in the original study (RVS05a), but the basic procedure will be outlined here; the method uses assumptions similar to those in a curve of growth analysis but does explicitly fit the line profiles. The spectral extraction was done while keeping a constant physical aperture size of $\sim 3 \mathrm{kpc}$; this aperture size was selected to match the seeing and therefore reduce host galaxy contamination as much as possible. The reduction and calibration were performed using standard IRAF reduction packages. HeNeAr lamps were used for wavelength calibration and stars such as G191-B2B and BD+25 4655 were used as flux standards.

Once the spectra were reduced and calibrated, the region containing the Na I D absorption doublet, as well as the neighboring He I $\lambda 5876$ emission line, was isolated. A Levenberg-

\footnotetext{
${ }^{1}$ http: //nedwww.ipac. caltech.edu/index.html
} 
Marquardt fitting routine was used to fit one to two components to the He I emission and the Na I D absorption doublet line profile. For emission lines, the formula used to fit the intensity of the line was:

$$
I(\lambda)=1+A e^{\left(\left(\lambda-\lambda_{0}\right) c /\left(\lambda_{0} b\right)\right)^{2}}
$$

where the amplitude, A, the Doppler parameter, $b(b=\sqrt{2} \sigma)$, and the central wavelength, $\lambda_{0}$, were free parameters. For absorption lines, the formula used to fit was:

$$
I(\lambda)=1-C_{f}(\lambda)+C_{f}(\lambda) e^{-\tau_{1}(\lambda)-\tau_{2}(\lambda)}
$$

where $C_{f}$ is the covering fraction of the line (see Section 7.1.2), and $\tau_{1}$ and $\tau_{2}$ represent the optical depths of each of the two lines of the Na I D doublet; $\tau_{1}$ corresponds to the line at $5896 \AA$, and $\tau_{2}=2 \tau_{1}$. In general, the optical depth $\tau_{i}$ is given by:

$$
\tau_{i}(\lambda)=\tau_{i, 0} e^{-\left(\lambda-\lambda_{i, 0}\right)^{2} /\left(\lambda_{i, 0} b / c\right)^{2}}
$$

Equations 2 and 3 were used to fit each line of the Na I D doublet. For each Na I D doublet pair, the covering fraction, $C_{f}$, central optical depth and wavelength of the red line $\left(\tau_{1}\right.$ and $\lambda_{0,1}$ ), and line width, $b$, were free parameters in the fit. Covering fraction was assumed to be independent of wavelength (and the same for both doublet lines), and the optical depths of the two doublet lines are fixed by atomic physics (as stated above: $\tau_{2}=2 \tau_{1}$ ). Because of these constraints, and the assumption of a Gaussian in optical depth space, both $C_{f}$ and $\tau$ can be constrained simultaneously for each doublet. It should be noted, however, that there is still some anticorrelation between $C_{f}$ and $\tau$ in the fit due to uncertainty in line shape and relative intensities. In the case of multiple, Gaussian velocity components, each velocity component arises in both lines of the doublet, so that $C_{f}$ and $\tau$ can be constrained independently for each velocity component.

Initial guesses for these parameters were supplied to the fitting program and a best fit was determined and plotted. Either one or two components were used to fit the He I emission line. Unless an absorption fit with two separate velocity components (corresponding to two separate Na I D doublets) was considerably better than a fit with one velocity component, only one velocity component (one Na I D doublet) was used to fit the absorption features. Only six objects showed convincing evidence for a two velocity components in absorption. Examples of the deblending of the Na I D feature for two Seyfert 1 and two Seyfert 2 objects can be seen in Figure 1. After fitting was performed, a Monte Carlo simulation with 1000 iterations was then run, in order to determine the parameter errors.

Particular care is needed when fitting the He I emission at $5876 \AA$, since the He I emission line in these objects is generally quite broad and has multiple components. So, for those objects showing He I emission lines, the $\mathrm{H} \alpha+[\mathrm{N} \mathrm{II}]$ complex was also examined to verify 
the accuracy of the fit to He I . An interpolation script was written in IDL to subtract out the $[\mathrm{N} \mathrm{II}] \lambda \lambda 6548,6583$ features and thus isolate the $\mathrm{H} \alpha$ line. The resulting $\mathrm{H} \alpha$ line was generally fit using two components, one narrow and one broad. The He I + Na I D complex was then refit, but this time parameters for He I emission lines were constrained to be those of the $\mathrm{H} \alpha$ line. The results from the fits with fully fixed parameters ("fixed") were then compared to those from the fits with free-floating parameters ("free"); agreement is observed within 2-5\%. Again, the errors on these parameters were determined from the Monte Carlo simulations after all fits were completed. The reduced $\chi^{2}$ for the two types of fits are compared in Figure 2. There are fewer points farther away from a reduced $\chi^{2}$ of unity for the free-floating fit. We therefore choose to use the results from the free-floating fits in the following discussion (this is further justified in the next section).

\subsection{Velocities}

Once fitting was done, velocities for these Na I D absorption lines were calculated, relative to the systemic velocities using redshifts as outlined in Section 2.1. In the following, $\Delta v$ is the velocity difference between the $\mathrm{Na}$ I $\mathrm{D}$ velocity centroid measured in the fit and the systemic Na I D velocity. Errors were propagated through both from the fit and the redshift measurement. A "maximum" velocity of $\Delta v_{\max } \equiv \Delta v-\mathrm{FWHM} / 2$, which takes into account both the shift and width of the absorption line, was also computed. Values of $\Delta v$ and $\Delta v_{\max }$ were compared for values resulting from the free He I fit and the fixed He I fit in order to determine the impact of fixing the He I parameters to those of $\mathrm{H} \alpha$. Plots of those comparisons are displayed in Figure 3. The agreement is good, with only three objects showing values that are not equal within the uncertainties. Of those, only one has $\left|\Delta v_{\text {free }}-\Delta v_{\text {fixed }}\right|>50 \mathrm{~km} \mathrm{~s}^{-1}$, which would change its outflow or inflow classification. In all three of those outlying cases, the He I line appears to be broader than the $\mathrm{H} \alpha$ line. This is plausible, since all of these objects are Seyfert 1 galaxies and so collisional and radiative transfer effects in the broad line region may affect the He I profile in a different way than the $\mathrm{H} \alpha$ profile. As there is little significant difference between the free-floating and fully-fixed fits, our adoption of the free-floating fits in Section 4.1 seems appropriate.

After velocities were calculated, a detection criterion of $\Delta v<-50 \mathrm{~km} \mathrm{~s}^{-1}$ was used to determine whether or not an object possesses an outflow. Similarly, $\Delta v>50 \mathrm{~km} \mathrm{~s}^{-1}$ was used as the cutoff for the detection of an inflow. A $2-\sigma$ threshold in measurement uncertainty $(|\Delta v|>2 \delta(\Delta v)$, where $\delta(\Delta v)$ is the measurement error) was also required for inflow/outflow classification, but this did not eliminate any objects from being classified as showing inflow

or outflow. As outlined in the initial study (RVS05a), the $50 \mathrm{~km} \mathrm{~s}^{-1}$ cutoff accounts for 
potential contamination from errors in wavelength calibration $\left(\lesssim 10 \mathrm{~km} \mathrm{~s}^{-1}\right)$, fitting $(\sim 10$ $\left.\mathrm{km} \mathrm{s}^{-1}\right)$, redshift measurements $\left(\sim 10-15 \mathrm{~km} \mathrm{~s}^{-1}\right)$, and the possibility of small blue- and redshifts due to gas in rotation. Fit parameters and derived velocities are listed in Table 2 ,

\subsection{Column Densities}

The optical depth $\tau$ and Doppler parameter $b$ as determined by the fitting function were used to calculate column densities of $\mathrm{Na}$ I D and $\mathrm{H}$ along the line of sight. As described in Spitzer (1978), the column density of $\mathrm{Na}$ I D in $\mathrm{cm}^{-2}$ is given by:

$$
N(\mathrm{Na} \text { I D })=\frac{\tau_{1, c} b}{1.497 \times 10^{-15} \lambda_{1} f_{1}}
$$

where $f_{1}$ is the oscillator strength, $\lambda_{1}$ is the rest frame vacuum wavelength $(\AA)$, and $\tau_{1, c}$ is the central optical depth of the Na I D $\lambda 5896$ line. The Doppler parameter, $b$, is in units of $\mathrm{km} \mathrm{s}^{-1}$. Values of $f_{1}=0.3180$ and $\lambda_{1}=5897.55 \AA$ were taken from Morton (1991).

To properly calculate the column density of hydrogen, we must correct for the effects of dust depletion and ionization. This process is also outlined in RVS05a, and uses empirical results that assume Galactic depletion and an ionization fraction of 0.9. The formula used is:

$$
N(\mathrm{H})=N(\mathrm{Na} \mathrm{I})(1-y)^{-1} 10^{-(a+b)}
$$

Column densities are again given in $\mathrm{cm}^{-2}, y$ is the ionization fraction, and $a$ and $b$ are the $\mathrm{Na}$ abundance and depletion onto dust in the object for which calculations are being performed, respectively. Whereas a near-IR luminosity-metallicity relation was used to determine $\mathrm{Na}$ abundance in the previous RVS05b and RVS05c studies, we here assume that these objects have solar $\mathrm{Na}$ abundance. Calculated column densities for each object can be found in Table 2.

\subsection{Stellar Na I D Contribution}

Stellar Na I D absorption may contribute to the observed Na I D feature. Stellar features from other elements may also contaminate the measurements if they are located close to the Na I D feature. The original study used a scaling relation between Na I D and $\mathrm{Mg}$ I $b$ equivalent width to determine the stellar $\mathrm{Na}$ I D contribution, as $\mathrm{Na}$ and $\mathrm{Mg}$ are created in a similar fashion (RVS05a). However, the spectral range for our data begins at roughly $5500 \AA$ and so does not include that $\mathrm{Mg}$ I $b$ triplet, so we must use the other method 
outlined in that study: stellar population synthesis models. As in RVS05a, we used the population synthesis code (Sed@.02) of González Delgado et al. (2005), which combines a young (40 Myr), instantaneous burst stellar population with an old (10 Gyr) population. One model uses a stellar mass ratio of $10 \% / 90 \%$ for young versus old populations, and the other uses $1 \% / 99 \%$. In order to enhance weak stellar features, the spectra were boxcar smoothed by $150 \mathrm{~km} \mathrm{~s}^{-1}$ and convolved with a $\sigma=200 \mathrm{~km} \mathrm{~s}^{-1}$ Gaussian. Our spectra were emission subtracted, using fitting parameters to remove He I and leave only the $\mathrm{Na}$ I D absorption. They were then overlaid with the stellar population synthesis models.

Almost all objects with suspected outflows or inflows showed absorption that was much deeper than the stellar absorption alone. The extreme examples of Mrk 352 and Mrk 6 are shown in Figure 4. In Mrk 352, the results from stellar population synthesis show a high likelihood that the bulk of the $\mathrm{Na}$ I D absorption is stellar. In contrast, Mrk 6 shows a definite high-velocity outflow.

\section{RESULTS: OUTFLOWS}

\subsection{Seyfert 2}

Figure 5 shows the He I and Na I D complexes for all of the 17 Seyfert 2 galaxies in this study, plotted on a velocity scale based on Na I D $\lambda 5890$ systemic. Tables 3 and 4 list the measured properties for each $\mathrm{Na}$ I D outflow velocity component and overall outflow averages, combined with data for Seyfert ULIRGs from RVS05c.

Out of a total of 17 Seyfert 2 galaxies, we detected blueshifted Na I D absorption with $\Delta v<-50 \mathrm{~km} \mathrm{~s}^{-1}$ in 3 objects, for an outflow rate of $18 \pm 9 \%$. No objects were ruled out by the $2-\sigma$ criterion in measurement uncertainty, which requires $|\Delta v|>2 \delta(\Delta v)$ for a detection (see Section 4.2). Of those objects which fit the outflow criteria, only one, NGC 7319, showed convincing evidence for two velocity components in absorption. The implications of this low detection rate are discussed in Section 7.1 .

Velocities for these objects were all within $20 \mathrm{~km} \mathrm{~s}^{-1}$ of each other, ranging from -130 to $-148 \mathrm{~km} \mathrm{~s}^{-1}$. The maximum velocity, $\Delta v_{\max } \equiv \Delta v-\mathrm{FWHM} / 2$, showed a much larger range (due to the broader range of Doppler parameters), from -176 to $-504 \mathrm{~km} \mathrm{~s}^{-1}$, with NGC 7319 showing the largest $\Delta v_{\max }$ (Table 4). We further discuss these results in Section 7.1 .

\footnotetext{
${ }^{2}$ http://www.iaa.es/ rosa/ and http://www.iaa.es/ $\mathrm{mcs} / \mathrm{sed} @$
} 
Previous studies have shown that it is common for Seyfert 2 galaxies to have asymmetric emission lines with extended blue wings. These asymmetries are generally believed to be the result of outflowing gas in the narrow line region of the AGN, and outflowing gas in the NLR has been spatially resolved in both Seyfert 1 and Seyfert 2 galaxies (Walker 1968; Wilson \& Ulvestad 1987; Ebstein et al. 1989; Veilleux 1991a,b.,c; Ruiz et al. 2005). This emission line asymmetry, however, is not an unambiguous indicator of outflow in the way absorption is since one cannot differentiate between outflowing line-emitting material in front of the nucleus and infalling material on the far side of the nucleus. Blue-wing emission asymmetries can only be interpreted as outflow if we are absolutely sure we are not seeing the opposite side of the object (Rodríguez-Ardila et al. 2006). The primary focus of our paper is blueshifted $\mathrm{Na}$ I D absorption, but it is of interest to look for blue-wing emission line asymmetry in our Seyfert 2 galaxies to see if it is at all correlated with our findings on Na I D outflows. In RVS05c, $75 \%$ of Seyfert 2 nuclei showed blue emission-line asymmetry (BELA), compared to a $45 \% \mathrm{Na}$ I D outflow detection rate.

We have used [N II] $\lambda \lambda 6548,6583$ to look for BELA in our objects (the high-ionization [O III] $\lambda 5007$ line most commonly used in BELA detection is not within our wavelength range). The $[\mathrm{N} \mathrm{II}] \lambda \lambda 6548,6583$ lines were separated from $\mathrm{H} \alpha$ by taking the blue wing of the $[\mathrm{N} \mathrm{II}] \lambda 6548$ line and the red wing of the $[\mathrm{N} \mathrm{II}] \lambda 6583$ line, and plotting them together about $\Delta v=0$. The two wings were then scaled appropriately to match each other in intensity, and BELA determination was done by visual inspection. 5 out of 17 Seyfert 2 galaxies (29\%) showed evidence for BELA. The most obvious case of BELA is NGC 5252. Of those which show BELA, only one (NGC 7319) has been determined to have an Na I D outflow, and one (Mrk 348) actually shows a Na I D inflow (Section 6.1), though it is the least asymmetric of all the BELA objects. Those five objects, along with a comparison object with a Na I D outflow but no BELA (Mrk 622), are shown in Figure6. Another object (NGC 3786; not shown in the figure) shows prominent red-wing asymmetry; this object presents an Na I D outflow rather than an inflow. Overall, we find no obvious correlation between emission and absorption signatures of outflow, although the statistics are poor. Moreover, we cannot formally rule out the possibility that the difference in outflow and BELA detection rates is simply due to

our use of $[\mathrm{N} \mathrm{II}]$ as a BELA probe since BELAs are more often seen in high-ionization lines like [O III] than in low-ionization lines like [N II] (Veilleux 1991a).

\subsection{Seyfert 1}

Figure 7 shows the He I and Na I D complexes for all of the 18 Seyfert 1 galaxies in this study, plotted on a velocity scale relative to Na I D $\lambda 5890$ systemic. As for the Seyfert 
2 galaxies, Tables 3 and 4 list the measured properties for each $\mathrm{Na}$ I D outflow velocity component and overall outflow averages.

Only 1 of the 18 Seyfert 1 galaxies was found to have blueshifted absorption with $\Delta v$ $<-50 \mathrm{~km} \mathrm{~s}^{-1}$, and as with Seyfert 2 inflows, the $2-\sigma$ criterion did not eliminate any objects (Section 4.2). The outflow detection rate is therefore only $6 \pm 6 \%$.

Mrk 6 was the only Seyfert 1 galaxy determined to have an outflow. Two velocity components were detected, each with a velocity higher than those for the Seyfert 2 galaxies: one component with $-229 \mathrm{~km} \mathrm{~s}^{-1}$ and one with $-1024 \mathrm{~km} \mathrm{~s}^{-1}$, giving a $\Delta v_{\max }=-1037$ $\mathrm{km} \mathrm{s}^{-1}$ (Table 4). See Section 7.1 for a discussion of these results.

\section{RESULTS: INFLOWS}

While only a few objects in our sample showed blueshifted Na I D absorption, a large percentage of objects in each Seyfert group showed redshifted Na I D absorption. We consider this redshifted absorption to be an unambiguous indicator of inflow, in the same way that blueshifted absorption is an unambiguous outflow indicator, since the continuum source (in this case the galaxy nucleus) must be behind the absorber. This section describes the results on inflow detection rates and kinematics. Table 5 lists the measured properties of these inflows in individual objects. The implications of these results are discussed in Section 7.2 .

\subsection{Seyfert 2}

The same general detection criteria used for determining outflows were used for determining inflows: We required a redshifted absorption of $\Delta v>50 \mathrm{~km} \mathrm{~s}^{-1}$, along with the $2-\sigma$ criterion (Section 4.2). Out of the 17 Seyfert 2 galaxies, 6 showed Na I D inflow, for a detection rate of $35 \pm 11 \%$.

Velocities for these six objects spanned the range from 51 to $127 \mathrm{~km} \mathrm{~s}^{-1}$. The maximum velocity, where in the case of inflows $\Delta v_{\max } \equiv \Delta v+\mathrm{FWHM} / 2$, ranged from 155 to 352 $\mathrm{km} \mathrm{s}^{-1}$. The largest $\Delta v$ was measured for UGC 3995, although NGC 3362 has a slightly larger $\Delta v_{\max }$ than UGC 3995 due to the difference in Doppler parameter. The average properties of these inflows are listed in Table 6 and discussed further in Section $[7.2$. 


\subsection{Seyfert 1}

Of the 18 Seyfert 1 galaxies, 7 showed Na I D inflow, for a detection rate of $39 \pm 12 \%$, i.e. similar to that of the Seyfert 2s. Again, no objects were ruled out by the $2 \sigma$ criterion (Section 4.2).

The Seyfert 1 inflow velocities spanned a similar range to those of the Seyfert 2 velocities, from 67 to $138 \mathrm{~km} \mathrm{~s}^{-1}$. The Seyfert $1 \Delta v_{\max }$ values showed a broader range than that of Seyfert 2, from 169 to $507 \mathrm{~km} \mathrm{~s}^{-1}$, and while Mrk 1126 has the highest $\Delta v$, NGC 7603 has the highest $\Delta v_{\max }$. Again, the average inflow properties are listed in Table 6 and the results are discussed further in Section 7.2 .

\section{DISCUSSION}

\subsection{Outflows}

\subsubsection{Comparison with Previous Studies}

Since the purpose of this study is to determine whether starbursts or AGN are the primary mechanism behind galactic outflows, it is important to compare the results determined here to those of RVS05c for the ULIRG Seyferts - galaxies with both starburst and AGN. As seen in Table 4, the detection rates for the IR-faint Seyfert 2s and Seyfert 1s are only $18 \% \pm 9 \%$ and $6 \% \pm 6 \%$, respectively, but rates were as high as $45 \% \pm 11 \%$ and $50 \% \pm$ $20 \%$ for the IR-luminous Seyfert 2s and 1s (RVS05c). Our detection rates are even lower in comparison to non-Seyfert ULIRGs, since rates of $80 \% \pm 7 \%$ and $46 \% \pm 13 \%$ were measured for low- $z$ and high- $z$ ULIRGs in RVS05b, and a rate of $83 \%$ was reported in Martin (2005). Poststarburst galaxies have also been found to have high outflow detection rates on the order of $70 \%$ (Tremonti et al. 2007). The measured outflow detection rates in the IR-faint Seyfert galaxies are therefore considerably smaller than those of pure starbursting galaxies, poststarbursts, and AGN + starburst composites.

Previous observations have indicated that the outflow detection rate increases with infrared luminosity (RVS05b, Sato et al. 2009). Figure 8 shows the outflow detection rates of the IR-faint Seyferts in our sample as well as the the IR-luminous Seyferts and starburst ULIRGs and LIRGs of RVS05b and RVS05c as a function of $L_{\mathrm{FIR}}$. The increase in outflow detection rate with $L_{\mathrm{FIR}}$ is apparent, despite the limited range in $L_{\mathrm{FIR}}$. As $L_{\mathrm{FIR}}$ is correlated

with the star formation rate, this increase in the detection rate with $L_{\text {FIR }}$ suggests that star formation in all of these systems is the main driver of neutral outflows detected in $\mathrm{Na}$ I. 
Geometry could also be playing a role, since AGN winds with smaller opening angles (higher collimation) would reduce the detection rate (see Section 7.1.2).

Histograms showing the distributions of all (negative and positive) velocities in our current data as well as those of RVS05b and RVS05c provide another point of comparison (Figure 9). The left panel compares the velocities of IR-faint Seyfert galaxy components from this study to the Seyfert and starburst ULIRGs \& LIRGs of RVS05b and RVS05c. We can safely rule out rotation as being a primary cause for the motion of the Na I D gas based on the fact that we do not observe a symmetry about zero velocity in these histograms. Were rotation the dominant gas motion, we should observe roughly equal amounts of blueshifted and redshifted gas in each object and in the overall distributions shown in Figure 8, assuming the Na I D gas is distributed more or less symmetrically around the center of rotation and is unaffected by severe differential obscuration. Negative velocities are found in a much higher percentage of IR-luminous objects (Seyferts of RVS05c and starbursts of RVS05b) than IR-faint objects, again indicating that outflows are both stronger and more frequent on average in objects with high SFR. The right panel in Figure 9 combines together the IR-faint and IR-luminous Seyferts from this study and RVS05c and compares them to the starbursts of RVS05b. In terms of the negative velocities, the results for Seyfert 1 and Seyfert 2 galaxies are quite different, with Seyfert 2 galaxies showing similar outflow percentages to those of the ULIRGs \& LIRGs. This suggests a physical connection between the mechanisms that drive outflows in Seyfert 2s and starbursting galaxies and a physical difference between the mechanisms that cause outflows in Seyfert 1s and Seyfert 2s. Interestingly, studies of ionized gas outflows have not found such a dichotomy in velocities between Seyfert 1s and Seyfert 2s (e.g., Ruiz et al. 2001; Veilleux et al. 2005; Ruiz et al. 2005; Das et al. 2007, and references therein). This suggests that the neutral gas probed by our Na I D observations is not kinematically related to the ionized material of the NLR. This difference is even more obvious when we also consider our results on positive (inflow) velocities (Section 7.2.1).

Rigorous statistical tests confirm these results. Kolmogorov-Smirnov (K-S) and Kuiper tests were both used, as the K-S test has an inherent bias in terms of differences in the mean and the Kuiper test does not. Low values reported by both of these tests can help rule out two sets of data having the same parent distribution. Results of these tests are listed in Table 7. The small values of $P$ (null) for $\Delta v$ and $\Delta v_{\max }$ when comparing IR-faint and IR-luminous Seyferts suggest that they do not come from the same parent distribution. A more significant comparison comes from combining together the results for the IR-faint Seyfert galaxies from this study and those for the IR-luminous Seyfert galaxies from RVS05c, and comparing them with those for the starburst ULIRGs and LIRGs from RVS05b. The same statistical tests were performed on these distributions, first using all velocities, then the outflowing components only. The results are listed in Tables 8 and 9 , respectively. The results in Table 
8 indicate that Seyferts and starbursts do not share the same parent velocity distributions; we return to this point in Section 7.2. When considering only the outflowing components, all comparisons show low probability of originating from the same parent distribution except when the Seyfert 2 galaxies are compared with the starburst ULIRGs \& LIRGs. In that case, both the K-S and Kuiper tests return large $P$ (null) values. This confirms quantitatively that the outflows in these two classes of objects may arise from the same physical process.

Figure 10 displays plots showing $\Delta v_{\max }$ as a function of far-IR luminosity (correlated with star formation rate) and of galactic circular velocity (correlated with galactic mass) for Seyferts from this study, IR-luminous Seyferts from RVS05c, starburst LIRGs \& ULIRGs from RVS05b, and four starburst dwarf galaxies from Schwartz \& Martin (2004). The four dwarfs were added in order to see if low-mass galaxies follow the same trends as high-mass systems. The IR-faint Seyfert 2 galaxies seem to follow the same trends as the IR-bright galaxies, with $\Delta v_{\max }$ increasing with both SFR and galactic mass, while the outflow velocities of all Seyfert 1 galaxies lie above these trends. This again suggests a fundamental difference in the way the winds in IR-faint/bright Seyfert 1 galaxies are powered compared with the winds in IR-faint/bright Seyfert 2 and starburst galaxies. We return to this issue in Section 7.1.2.

\subsubsection{Outflow Dynamics}

Another useful comparison between IR-faint and IR-luminous Seyferts is to look at the dynamical properties in addition to the kinematics. Using calculated covering fraction, column density, and velocities, we can estimate the mass, momentum, and kinetic energy of the neutral gas phase of the ISM being probed by those winds. We follow the method outlined in the original study (RVS05b), which made the assumption that the outflows are spherically symmetric mass-conserving free winds, with a velocity and instantaneous mass outflow rate which do not depend on radius within the wind and which are zero outside the wind. This method also assumed that the wind is a thin shell with a uniform radius

of $5 \mathrm{kpc}$. This value was based on actual spatial measurements in some of the objects of RVS05c, but such spatial measurements are not available for our IR-faint Seyfert galaxies. In the present study, we use a $5 \mathrm{kpc}$ radius for both the Seyfert 2 and Seyfert 1 galaxies. This is different from the radius of $10 \mathrm{pc}$ that was used for the Seyfert 1 galaxies in the original study (RVS05c); the radius in IR-faint Seyfert 1s was chosen to be the same as for IR-faint Seyfert $2 \mathrm{~s}$ to facilitate comparisons within the IR-faint sample, and to that end, the IR-luminous Seyfert 1 dynamical values from RVS05c have been scaled up to a $5 \mathrm{kpc}$ radius as well. These results should be considered order-of-magnitude estimates since they 
are based on a number of largely unproven assumptions. We have not listed all results here; selected results can be seen in Figure 11.

For the objects in the present study, we assume a modest value of 0.3 for the largescale opening angle $\left(C_{\Omega}\right)$, which is the typical value for local disk winds (Veilleux et al. 2005). Following the method of RVS05b, the covering fraction is used to parameterize the clumpiness of the wind, or it may reflect the global solid angle subtended by the wind when viewed from the galactic center. Using the value of $\left\langle C_{f}\right\rangle$ as listed in Table 4 , this yields a global covering factor of $\Omega \sim 0.1$ for both Seyfert 1 s and Seyfert 2s. These values are not well constrained, especially for the Seyfert 1 s since only one outflow was detected in this type of Seyfert. The $\Omega$ value is inconsistent with values of $\Omega \sim 0.5-1.0$ calculated by Crenshaw et al. (2003b) from UV absorption lines in local Seyfert 1 s. This low $\Omega$ value could imply that the winds we are seeing are collimated rather than wide-angle, and thus our lack of outflow detection in some of these objects may be due to their orientation relative to us rather than a complete lack of neutral gas outflow. The influence of host galaxy inclination on column density was explored using extinction-corrected axis ratios from the de Vaucouleurs Third Reference Catalog (?), but no conclusion could be drawn due to small-number statistics. Additionally, these $\Omega$ values will be low in comparison to values from other studies; there is contribution here from the background galaxy, and many of the outflows we have found are small-scale. Comparisons with the results of UV absorption line studies should be considered with caution, though, since UV absorbers are typically of much higher ionization (N V, C IV) and likely located much closer to the AGN than Na I D absorbing material. The Na I D absorbers may also be affected more strongly by host galaxy contamination than the UV absorbers. Blueshifted absorption lines have also been detected in X-ray spectra for a number of Seyfert galaxies, including Mrk 6, with column densities of significantly higher order of magnitude than we have found here for Na I D in the optical (e.g., Mathur et al. 1995; Feldmeier et al. 1999; Monier et al. 2001; Krongold et al. 2003; Vaughan \& Uttley 2008). Again, we caution comparing these results with our own, as it has been noted that these X-ray lines are much higher ionization (O VII, O VIII) and are often intrinsically related to the aforementioned UV absorption lines.

One particularly interesting dynamical quantity is the mass outflow rate, since galactic outflows may contribute to the IGM enrichment and are possibly a quencher of star formation (Tremonti et al. 2007). Plots of $d M / d t$ for the IR-faint Seyferts, IR-luminous Seyferts, and starbursting LIRGs and ULIRGS from RVS05b and RVS05c, and the dwarfs of Schwartz \& Martin (all calculations are based on a absorber radius of $5 \mathrm{kpc}$ ) are presented in Figure 11, We see a general trend of mass outflow rate increasing with both $L_{\text {FIR }}$ and galactic mass. There is a considerable difference in the mean $d M / d t$ rates between IR-luminous Seyfert 1s and IR-luminous Seyfert 2s. The momentum and energy, as well as outflow rates for those 
quantities, are also significantly higher for IR-luminous Seyfert 1s than IR-luminous Seyfert 2s and all IR-faint Seyferts. The overall good agreement in detection rate and kinematics between IR-faint and IR-bright Seyfert 2s and starburst ULIRGs \& LIRGs (Figures 8, 11) suggests that the outflows in all of these objects are powered by star formation, while the marked differences when IR-faint Seyfert 1 galaxies are considered suggest that the AGN plays an important role in driving the (generally high velocity) outflows in Seyfert 1 galaxies. Escape fractions were not calculated here since too few objects have outflow velocity components.

We can also look at energy outflow rates in order to determine what role these outflows could play in galactic feedback. The rates that we have calculated can be found in Table 3 , though it must be cautioned that these numbers are a function of our uncertain $\Omega$ value, as well as our assumed absorber radius of $5 \mathrm{kpc}$. For the Seyfert 2 outflows, the average energy outflow rate was found to be $\sim 10^{41.1}\left(\frac{\Omega}{0.1}\right)\left(\frac{r}{5 \mathrm{kpc}}\right) \mathrm{ergs} \mathrm{s}^{-1}$, or $\sim 10^{7}\left(\frac{\Omega}{0.1}\right)\left(\frac{r}{5 \mathrm{kpc}}\right) L_{\odot}$. In comparison to the average bolometric luminosity for these objects $\left(\sim 10^{10} L_{\odot}\right.$, taken from Woo \& Urry 2002), the energy outflow rates are only $\sim 1 \%$ of the host galaxy luminosity. This indicates that outflow energetics in our Seyfert 2 galaxies are not strong enough to play a large role in galactic feedback. The findings of Schlesinger et al. (2009) for Mrk 573 are in agreement with our conclusion. For the only Seyfert 1 galaxy that we have found to have an outflow, Mrk 6, the energy outflow rate of its lower velocity component $\left(10^{41.6}\right.$

$\left.\left(\frac{\Omega}{0.1}\right)\left(\frac{r}{5 \mathrm{kpc}}\right) \operatorname{ergs~s}^{-1}\right)$ is again $\sim 1 \%$ of the host bolometric luminosity. This indicates that this Seyfert 1 outflow is also not energetic enough to play a vital role in galactic feedback, and thus previous findings are consistent with our results (Krongold et al. 2007; Stoll et al. 2009). If we look at the higher velocity outflow found in Mrk 6, its energy outflow rate $\left(10^{42.9}\left(\frac{\Omega}{0.1}\right)\left(\frac{r}{5 \mathrm{kpc}}\right) \mathrm{ergs} \mathrm{s}^{-1}\right)$ is $\sim 5 \%$ of the host bolometric luminosity, which is higher than for the Seyfert 2 galaxies but again likely not strong enough to influence the evolution of its environment. However, one should note that these values are all highly dependent on the calculated value of $\Omega$ and assumed value of $r$ and are thus uncertain in comparison to our measured velocities.

\subsection{Inflows}

\subsubsection{Comparison with Previous Studies}

An unexpected result of the present study is the high detection rate of inflows in IR-faint Seyferts $(39 \% \pm 12 \%$ for Seyfert 1s, $35 \% \pm 11 \%$ for Seyfert $2 \mathrm{~s})$. In contrast, only $\sim 15 \%$ of the IR-luminous objects in RVS05b and RVS05c showed redshifted Na I D absorption. This difference is clearly seen in the left panel of Figure 9. Interestingly, a recent search for 
outflows in the AEGIS database has also revealed an excess of inflows among AGN-powered systems (Sato et al. 2009). Inflow has been observed in at least one Seyfert 1 galaxy, NGC 5548 , using ionized gas detected in the UV, though detections have not been reported for such a large number of objects as we have found here (Mathur et al. 1999). There has been one tentative observation of redshifted X-ray absorption in a Seyfert 1 galaxy, but the authors caution that the significance of the absorption line they have measured is highly uncertain (Dadina et al. 2005).

When we examine the inflow data on the IR-faint galaxies alone, we find no significant correlation between $\Delta v_{\max }$ and galactic mass (left panel in Figure 12). Neither is there any obvious trend with Seyfert type or far-infrared luminosity (right panel of Figure 12), in contrast to the trends seen for the outflowing gas. Additionally, we see inflows in nearly the same fraction of Seyfert 1s and Seyfert 2s, indicating no particular trend with Seyfert type. As for the detected outflows, the influence of host galaxy inclination on column density was explored using extinction-corrected axis ratios (?), but again, no conclusion could be drawn due to small-number statistics.

Next, the velocity distributions of the inflowing components for the IR-faint and IRluminous Seyfert 1 galaxies were combined together and compared with the combined distribution of IR-faint and IR-luminous Seyfert 2 galaxies. K-S and Kuiper tests were performed on $\Delta v, \Delta v_{\max }$, and Doppler parameter, following the same procedure as in Section 7.1, and the results are listed in Table 10. They confirm the lack of obvious differences in the inflow properties between the two types of Seyfert galaxies.

\subsubsection{Search for Connection between Inflows and Nuclear Structures}

The Na I absorption infall velocities often extend to relatively high values so we favor a nuclear location for this gas rather than a galactic origin (e.g. galactic fountains, Ferrara \& Ricotti 2006). Nuclear accretion of cool gas like Na I with T $\sim 100 \mathrm{~K}$ can provide fuel not only for star formation but also for nuclear activity (Struve et al. 2008). Various mechanisms have been proposed to help reduce the large angular momentum of the gas in the nuclei of galaxies. These include nuclear $(\lesssim 1 \mathrm{kpc})$ bars and spirals (Pogge \& Martini 2002; Martini et al. 2003a,b; ?) and gravitational interactions with neighboring galaxies (Crenshaw et al. 2003a). Nuclear bars are thought to form when a large galactic bar forces gas inwards, creating a gaseous disk, and instability causes formation of a small gas bar near the nucleus (Shlosman et al. 1989; Martini et al. 2001). Stellar bars could also accomplish the same thing. Both bar types are capable of removing angular momentum from gas rotating

near the nucleus (Martini et al. 2001). Nuclear spirals have been proposed as another AGN 
fueling mechanism since shock fronts that occur at their boundaries can take away angular momentum from local gas and cause material to fall in towards the black hole (Martini et al. $2003 \mathrm{~b})$. However, these nuclear structures do not necessarily lead to AGN fueling since they are present in a equally large fraction of non-active galaxies (Pogge \& Martini 2002). Star formation may occur in these objects and disrupt AGN-fueling inflows (?).

We revisit this issue here by looking for the presence of nuclear spirals or bars in the IR-faint Seyfert galaxies with inflow signature. We have compared our results to nuclear structure surveys done by three different groups (Malkan et al. 1998; Martini et al. 2003a; Deo et al. 2006). Nuclear dust structures in objects in common with these studies are classified into five distinct morphological categories (see Column (11) of Table 1): irregular dust, dust filaments, nuclear dust ring, nuclear dust bar, and nuclear dust spiral. Of the thirteen objects which show inflows, five show evidence for nuclear dust spirals, bars, or rings, one shows evidence for dust filaments, one for irregular dust, and six show no sign of nuclear dust structure. The significant fraction of objects that show both inflows and nuclear dust spirals, bars, rings, and/or filaments lends credence to the idea of a connection between morphology and kinematics. Of the seven objects with irregular dust or no dust, five have nearby companion galaxies, so tidal forces due to interactions with these companions may cause AGN fueling (Rafanelli et al. 1995; Henriksen \& Cousineau 1999; Smith et al. 2007). This leaves only two objects with inflow which do not show nuclear structure or a companion: Akn 202 and Mrk 1018. There are also two objects in our study with nuclear dust structure that show measurable outflow rather than inflow (NGC 3786 and NGC 7319), and thus whether we measure outflow rather than inflow may be a consequence of our line of sight to the nucleus (inflow and outflow may be occurring in different planes, or in the same plane but over a different range of azimuthal angles), rather than a lack of inflow in the object. Measurements of the line-of-sight velocity field with a resolution of $\sim 10$ s of pc will be needed to disentangle the geometry of the inflows/outflows detected in our data (e.g., the study of NGC 1097 by ?van de Ven \& Fathi 2009).

\subsubsection{Inflow Dynamics}

The same method used to calculate mass, momentum, and kinetic energy for outflows (Section 7.1.2) was used for inflows, but the characteristic absorber radius was reduced to 1 kpc, a rough upper limit to the scale of the circumnuclear structures (nuclear bars/spirals) believed to be responsible for feeding the AGN (Section 7.2.2, Martini et al. 2003a). Again, these dynamical quantities are rather uncertain, but are calculated to find out at least roughly how these inflows compare with the mass accretion rates necessary to power the 
AGN in these systems. Table 5 lists the mass, momentum, and kinetic energy calculated for all objects with measured inflows. The mass accretion rate for these inflows ranges from just under $1\left(\frac{r}{1 \mathrm{kpc}}\right) \mathrm{M}_{\odot} \mathrm{yr}^{-1}$ to just under $5\left(\frac{r}{1 \mathrm{kpc}}\right) \mathrm{M}_{\odot} \mathrm{yr}^{-1}$. For comparison, the mass accretion rate needed to power an AGN is $\dot{M}=L_{\mathrm{bol}} / c^{2} \eta$, where $L_{b o l}$ is the bolometric luminosity of the AGN, $\dot{M}$ is the mass accretion rate, $c$ is the speed of light, and $\eta$ is an efficiency factor dictating how much of the rest mass of the material being accreted is turned into radiation. If we take $\eta$ to be $\approx 0.1$ (Riffel et al. 2008) and allow the bolometric luminosity to be $\sim 10^{44} \mathrm{ergs} \mathrm{s}^{-1}$, typical for Seyfert galaxies (Padovani \& Rafanelli 1988; Crenshaw et al. 2003b), then we find that a mass accretion rate of $\dot{M} \sim 10^{-2} \mathrm{M}_{\odot} \mathrm{yr}^{-1}$ is required. Even with our rough order of magnitude estimates, the mass accretion rates of all of our observed $\mathrm{Na}$ I D inflows are well above the amount necessary to power the AGN. Thus the inflows that we are measuring carry enough material to fuel the AGN in these objects, even if only $\sim 1 \%$ of this material makes its way down to the AGN. The total infalling mass of $\sim 10^{7}$ $\left(\frac{r}{1 \mathrm{kpc}}\right) M_{\odot}$, estimated from our data, is enough to sustain nuclear activity over typical AGN lifetimes ( $10^{7}-10^{8} \mathrm{yrs}$; Mouri \& Taniguchi 2002; ?).

\section{SUMMARY}

The main results from our study of Na I D absorption in infrared-faint Seyfert 1 and Seyfert 2 galaxies can be summarized as follows:

- Outflow Detection Rates and Kinematics: The rates of detection of outflows in IR-faint Seyfert 1 and 2 galaxies are lower than previously found in IR-bright Seyferts. Outflows were found in $\sim 18 \%$ of IR-faint Seyfert $2 \mathrm{~s}$ in our sample, compared with $\sim 45 \%$ among the Seyfert 2 ULIRGs of RVS05c. Only one out of 18 Seyfert 1 galaxies in our sample shows evidence for a wind, far lower than the $\sim 50 \%$ reported for Seyfert 1 ULIRGs in RVS05c. Interestingly, the outflow detection rate and velocities of IR-faint Seyferts follow the same trends with infrared luminosity and galaxy mass as IR-bright systems. The outflow kinematics of Seyfert 2 galaxies resemble those of starburst galaxies, while the outflow velocities in Seyfert 1 galaxies are significantly larger. These results suggest that the AGN is contributing to the neutral outflows in Seyfert 1 systems, while the starburst is the main driver of the outflows in all Seyfert 2 galaxies. Differences in wind angular extent (e.g. AGN-driven outflows in Seyfert 1s are more collimated than starburst-driven outflows) may also explain some of these results.

- Outflow Dynamical Estimates: The mass, momentum, and kinetic energy of the material involved in these outflows were estimated assuming a constant characteristic radius 
of $5 \mathrm{kpc}$. The dynamical properties of the outflows in Seyfert 2 galaxies are similar to those of the starburst ULIRGs \& LIRGs, but differ significantly from those of the Seyfert 1s. This again suggests a fundamental physical difference between the outflows of Seyfert 1s and those in the other objects. An attempt to determine the influence of host galaxy inclination on these outflows was inconclusive. Measured energetic rates do not appear large enough to play a significant role in galactic feedback, but these values are uncertain.

- Inflow Detection Rates and Kinematics: A striking result of this study is the high rate of detection of spatially-unresolved redshifted Na I D absorption, which we interpret as nuclear inflows (39\% and 35\% inflow detection rates for Seyfert $2 \mathrm{~s}$ and 1s), with maximum velocities (321 and $291 \mathrm{~km} \mathrm{~s}^{-1}$ on average). This is evidence for the existence of some mechanism capable of removing angular momentum from the circumnuclear gas in these objects. Nuclear bars and spirals, as well as interactions with nearby galaxies, may play a role in this process.

- Inflow Dynamical Estimates: Mass, momentum, and kinetic energy were estimated for the inflows, using a characteristic radius of $1 \mathrm{kpc}$, consistent with the observed sizes of nuclear bars and spirals in these systems. While these estimates are uncertain, we find that the total infalling mass and infalling mass rates are more than enough to power the AGN in these systems for typical AGN lifetimes. As with the outflows, an attempt was made to determine the influence of inclination on column density, but no conclusion could be drawn. 
H.B.K., D.S.N.R., and S.V. were supported in part by NSF through contract AST/EXC 0606932. S.V. also acknowledges support from a Senior Award from the Alexander von Humboldt Foundation and thanks the host institution, MPE Garching, where some of this work was performed. H.B.K. would like to acknowledge Stacy McGaugh and Massimo Ricotti for helpful discussions during the writing process. The authors would also like to thank Michael McDonald for his assistance with Perl computation code. The observations reported here were obtained at the Kitt Peak National Observatory, National Optical Astronomy Observatory, which is operated by the Association of Universities for Research in Astronomy (AURA), Inc., under cooperative agreement with the National Science Foundation. This research has made use of the NASA/IPAC Extragalactic Database (NED), which is operated by JPL/Caltech, under contract with NASA. 


\section{REFERENCES}

Binney, J., Gerhard, O., \& Silk, J. 2001, MNRAS, 321, 471

Binney, J. 2004, MNRAS, 347, 1093

Cole, S., Lacey, C. G., Baugh, C. M., \& Frenk, C. S. 2000, MNRAS, 319, 168

Cooper, J., Bicknell, G., Sutherlan, R., \& Bland-Hawthorn, J. 2008, ApJ, 674, 157

Crenshaw, D. M., Kraemer, S. B., \& Gabel, J. R. 2003a, AJ, 126, 1690

Crenshaw, D. M., Kraemer, S. B., \& George, I. M. 2003b, ARA\&A, 41, 117

Croom, S., et al. 2004, AGN Physics with the Sloan Digital Sky Survey Conf. Proc., eds G. T. Richards and P. B. Hall (San Francisco: ASP), 311, 457

Croston, J. H., Hardcastle, M. J., Kharb, P., Kraft, R. P., \& Hota, A. 2008, ApJ, 688, 190

Dadina, M., Cappi, M., Malaguti, G., Ponti, G., \& de Rosa, A. 2005, A\&A, 442, 461

Das, V., Crenshaw, D. M., \& Kraemer, S. B. 2007, ApJ, 656, 699

Davies, R. I., Maciejewski, W., Hicks, E. K. S., Tacconi, L. J., Genzel, R., \& Engel, H. 2009, ApJ, 702, 114

Deo, R. P., Crenshaw, D. M., \& Kraemer, S. B. 2006, AJ, 132, 321

Ebstein, S. M., Carleton, N. P., \& Papaliolios, C. 1989, ApJ, 336, 103

Feldmeier, J. J., Brandt, W. N., Elvis, M., Fabian, A. C., Iwasawa, K., \& Mathur, S. 1999, ApJ, 510, 167

Ferrara, A., \& Ricotti, M. 2006, MNRAS, 373, 571

Ferrarese, L., \& Merritt, D. 2000, ApJ, 539, L9

Garnett, D. R. 2002, ApJ, 581, 1019

González Delgado, R. M., Cervino, M., Martins, L. P., Leitherer, C., \& Hauschildt. P. H. 2005, MNRAS, 357, 945

Granato, G. L., De Zotti, G., Silva, L., Bressan, A., \& Danese, L. 2004, ApJ, 600, 580

Henriksen, M., \& Cousineau, S. 1999, ApJ, 511, 595 
Hota, A., \& Saikia, D. J. 2006, MNRAS, 371, 945

Irwin, J. A., \& Saikia, D. J. 2003, MNRAS, 346, 977

Kennicutt, R. C. 1998, ApJ, 498, 541

Kormendy, J., \& Kennicutt, R. C., Jr. 2004, ARA\&A, 42, 603

Krongold, Y., Nicastro, F., Brickhouse, N. S., Elvis, M., Liedahl, D. A., \& Mathur, S. 2003, ApJ, 597, 832

Krongold, Y., Nicastro, F., Elvis, M., Brickhouse, N., Binette, L., Mathur, S., \& JiménezBailón, E. 2007, ApJ, 659, 1022

Larson, R. B. 1974, MNRAS, 169, 229

Malkan, M. A., Gorjian, V., \& Tam, R. 1998, ApJS, 117, 25

Martin, C. L. 2005, ApJ, 621, 227

Martini, P., Pogge, R. W., Ravindranath, S., \& An, J. H. 2001, ApJ, 562, 139

Martini, P., Regan, M. W., Mulchaey, J. S., \& Pogge, R. W. 2003a, ApJS, 146, 353

Martini, P., Regan, M. W., Mulchaey, J. S., \& Pogge, R. W. 2003b, ApJ, 589, 774

Mathur, S., Elvis, M., \& Wilkes, B. 1995, ApJ, 452, 230

Mathur, S., Elvis, M., \& Wilkes, B. 1999, ApJ, 519, 605

McLure, R. J., \& Dunlop, J. S. 2002, MNRAS, 331, 795

Monier, E. M., Mathur, S., Wilkes, B., \& Elvis, M. 2001, ApJ, 559, 675

Morton, D. C. 1991, ApJS, 77, 119

Mouri, H., \& Taniguchi, Y. 2002, ApJ, 565, 786

Nelson, C. H. \& Whittle, M. 1995, ApJS, 99, 67

Netzer, H., et al. 2007, ApJ, 666, 806

Padovani, P., \& Rafanelli, P. 1988, A\&A, 205, 53

Pogge, R. W. \& Martini, P. 2002, ApJ, 529, 624

Rafanelli, P., Violato, M., \& Baruffolo, A. 1995, AJ, 109, 1546 
Riffel, R. A., Storchi-Bergmann, T., Winge, C., McGregor, P. J., Beck, T., \& Schmitt, H. 2008, MNRAS, 385, 1129

Rodríguez-Ardila, A. Prieto, M. A., Viegas, S., \& Gruenwald, R. 2006, ApJ, 653, 1098

Ruiz, J. R., Crenshaw, D. M., Kraemer, S. B., Bower, G. A., Gull, T. R., Hutchings, J. B., Kaiser, M. E., \& Weistrop, D. 2001, AJ, 122, 2961

Ruiz, J. R., Crenshaw, D. M., Kraemer, S. B., Bower, G. A., Gull, T. R., Hutchings, J. B., Kaiser, M. E., \& Weistrop, D. 2005, AJ, 129, 73

Rupke, D. S., Veilleux, S., \& Sanders, D. B. 2005a, ApJS, 160, 87 (RVS05a)

Rupke, D. S., Veilleux, S., \& Sanders, D. B. 2005b, ApJS, 160, 115 (RVS05b)

Rupke, D. S., Veilleux, S., \& Sanders, D. B. 2005c, ApJ, 632, 751 (RVS05c)

Sanders, D. B., \& Mirabel, I. F. 1996, ARA\&A, 34, 749

Sato, T., Martin, C. L., Noeske, K. G., Koo, D. C., \& Lotz, J. M. 2009, ApJ, 696, 214

Scannapieco, E., Silk, J., \& Bouwens, R. 2005, ApJ, 635, L13

Schiano, A. V. R. 1985, ApJ, 299, 24

Schlesinger, K., Pogge, R. W., Martini, P., Shields, J. C., \& Fields, D. 2009, ApJ, 699, 857

Schwartz, C. M., \& Martin, C. L. 2004, ApJ, 610, 201

Schweitzer, M., et al. 2006, ApJ, 649, 79

Shapley, A. E., Steidel, C. C., Pettini, M., \& Adelberger, K. L. 2003, ApJ, 588, 65

Shlosman, I., Frank, J., \& Begelman, M. C. 1989, Nature, 338, 45

Smith, B. J., Struck, C., Hancock, M., Appleton, P. N., Charmandaris, V., \& Reach, W. T. 2007, AJ, 133, 791

Somerville, R. S., \& Primack, J. R. 1999, MNRAS, 310, 1087

Spitzer, L. 1978, Physical Processes in the Interstellar Medium (New York: WileyInterscience)

Springob, C. M., et al. 2005, ApJS, 160, 149

Stoll, R., Mathur, S., Krongold, Y., \& Nicastro, F. 2009, arXiv:0903.5310 
Struve, C., Morganti, R., \& Oosterloo, T. A. 2008, MmSAI, 79, 1096

Thacker, R. J., Scannapieco, E., \& Couchman, H. M. P., 2006, ApJ, 653, 86

Tremonti, C. A., et al. 2004, ApJ, 613, 898

Tremonti, C. A., Moustakas, J., \& Diamond-Stanic, A. M. 2007, ApJ, 663, L77

van de Ven, G., \& Fathi, K. 2009, arXiv:0905.3556

de Vaucouleurs, G., de Vaucouleurs, A., Corwin, H. G., Buta, R. J., Paturel, G., \& Fouque, P. 1995, VizieR Online Data Catalog, 7155

Vaughan, S., \& Uttley, P. 2008, MNRAS, 390, 421

Veilleux, S. 1991a, ApJS, 75, 357

Veilleux, S. 1991b, ApJS, 75, 383

Veilleux, S. 1991c, ApJ, 369, 331

Veilleux, S., Cecil, G., \& Bland-Hawthorn, J. 2005, ARA\&A, 43, 769

Walker, M. F. 1968, ApJ, 151, 71

Wegner, G., et al. 2003,AJ, 126, 2268

Weiner, B. J., et al. 2009, ApJ, 692, 187

Whittle, M. 1992a, ApJS, 79, 49

Wilson, A. S., \& Ulvestad, J. S. 1987, ApJ, 319, 105

Woo, J.-H., \& Urry, C. M. 2002, ApJ, 579, 530

This preprint was prepared with the AAS IATEX macros v5.2. 
Table 1. Galaxy properties and observing logs

\begin{tabular}{|c|c|c|c|c|c|c|c|c|c|c|c|}
\hline $\begin{array}{l}\text { Name } \\
(1)\end{array}$ & $\begin{array}{c}z \\
(2)\end{array}$ & $\begin{array}{c}L_{\mathrm{IR}} \\
(3)\end{array}$ & $\begin{array}{l}L_{\mathrm{FIR}} \\
(4)\end{array}$ & $\begin{array}{l}\text { SFR } \\
(5)\end{array}$ & $\begin{array}{l}v_{c} \\
(6)\end{array}$ & $\begin{array}{l}W_{e q} \\
(7)\end{array}$ & $\begin{array}{c}\text { Run } \\
(8)\end{array}$ & $\begin{array}{c}t_{\text {exp }} \\
(9)\end{array}$ & $\begin{array}{l}\text { PA } \\
(10)\end{array}$ & $\begin{array}{c}\text { Structure } \\
\text { (11) }\end{array}$ & $\begin{array}{l}\text { Refs } \\
(12)\end{array}$ \\
\hline \multicolumn{12}{|c|}{ Seyfert 2s } \\
\hline Akn 79 & 0.01743 & $\cdots$ & $\cdots$ & $\cdots$ & 231 & 3.43 & 2005 Sep 05 & 4500 & 0 & - & 1 \\
\hline Arp 107A & 0.03463 & 10.63 & 10.09 & 2.14 & 308 & 2.20 & 2006 Apr 02 & 5400 & 0 & - & 1 \\
\hline Mrk 348 & 0.01516 & 10.40 & 9.89 & 1.33 & 191 & 1.53 & 2006 Nov 23 & 4800 & 0 & - & 1 \\
\hline Mrk 622 & 0.02347 & 10.72 & 10.26 & 3.15 & 301 & 2.03 & 2006 Apr 02 & 5400 & 0 & I & $1,4,7$ \\
\hline Mrk 686 & 0.01420 & 10.04 & 9.64 & 0.76 & 558 & 2.98 & 2006 Mar 31 & 4500 & 90 & $\mathrm{~S}$ & $1,5,6$ \\
\hline Mrk 1157 & 0.01510 & 10.41 & 10.13 & 2.32 & 585 & 1.99 & 2005 Sep 04 & 3540 & 0 & $\mathrm{R}$ & $1,4,7$ \\
\hline NGC 1358 & 0.01351 & 10.35 & 9.37 & 0.41 & 299 & 3.73 & 2006 Nov 21 & 4800 & 0 & - & 1 \\
\hline NGC 1667 & $0.01517^{\mathrm{a}}$ & 10.97 & 10.66 & 7.84 & 580 & 3.99 & 2006 Nov 21 & 4800 & 0 & S & $1,4,6$ \\
\hline NGC 3362 & 0.02767 & $\ldots$ & $\ldots$ & $\ldots$ & 358 & 1.92 & 2006 Mar 31 & 4500 & 90 & $\mathrm{~F}$ & 1,7 \\
\hline NGC 3786 & 0.00903 & $\ldots$ & $\ldots$ & $\cdots$ & 367 & 3.11 & 2006 Apr 02 & 5400 & 22 & $\mathrm{R}, \mathrm{S}$ & $1,6,8$ \\
\hline NGC 4388 & 0.00839 & 10.41 & 10.34 & 3.78 & 414 & 2.27 & 2006 Apr 02 & 5400 & 0 & I & $1,5,6$ \\
\hline NGC 5252 & 0.02308 & 10.39 & 9.84 & 1.18 & 340 & 3.72 & 2006 Apr 02 & 5400 & 0 & $\mathrm{R}, \mathrm{S}$ & $1,6,7$ \\
\hline NGC 5728 & $0.00932^{\mathrm{a}}$ & 10.60 & 10.32 & 3.63 & 434 & 2.25 & 2006 Apr 02 & 3600 & 0 & - & 2,4 \\
\hline NGC 7319 & 0.02236 & 10.56 & 10.21 & 2.83 & 210 & 6.61 & 2005 Sep 08 & 3240 & 0 & $\mathrm{~F}$ & 1,7 \\
\hline NGC 7672 & 0.01348 & 9.91 & 9.64 & 0.76 & 363 & 1.39 & 2006 Nov 21 & 7200 & 0 & - & 1,4 \\
\hline NGC 7682 & 0.01707 & $\ldots$ & $\ldots$ & $\ldots$ & 412 & 2.06 & 2006 Nov 21 & 6000 & 0 & S & 1,6 \\
\hline UGC 3995 & 0.01575 & 10.32 & 9.76 & 0.99 & 250 & 3.25 & 2006 Mar 31 & 4800 & 90 & - & 1,4 \\
\hline \multicolumn{12}{|c|}{ Seyfert 1s } \\
\hline Akn 202 & $0.02872^{\mathrm{b}}$ & 10.71 & 10.15 & 2.43 & 610 & 0.44 & 2006 Nov 23 & 6000 & 0 & - & 4 \\
\hline Akn 564 & $0.02468^{\mathrm{a}}$ & 10.75 & 10.14 & 2.40 & 443 & 0.00 & 2005 Sep 08 & 2040 & 0 & - & 2,5 \\
\hline Mrk 6 & $0.01951^{\mathrm{a}}$ & 10.59 & 10.09 & 2.14 & 593 & 1.08 & 2006 Nov 21 & 6000 & 0 & I & $1,4,7$ \\
\hline Mrk 10 & 0.02925 & 10.78 & 10.38 & 4.14 & 620 & 2.18 & 2006 Nov 21 & 3600 & 0 & - & 1 \\
\hline Mrk 79 & $0.02221^{\mathrm{a}}$ & 10.85 & 10.33 & 3.68 & 356 & 0.39 & 2006 Nov 22 & 4800 & 0 & S & $1,4,8$ \\
\hline Mrk 110 & $0.03513^{\mathrm{b}}$ & $\ldots$ & $\ldots$ & $\ldots$ & 147 & 0.00 & 2006 Nov 22 & 3600 & 0 & - & - \\
\hline Mrk 352 & $0.01486^{\mathrm{a}}$ & $\ldots$ & $\cdots$ & $\cdots$ & 300 & 0.60 & 2006 Nov 22 & 7200 & 0 & - & 2 \\
\hline Mrk 359 & 0.01694 & 10.35 & 9.96 & 1.59 & 235 & 0.55 & 2005 Sep 05 & 2700 & 0 & $\mathrm{~S}$ & $2,5,8$ \\
\hline Mrk 382 & $0.03348^{\mathrm{b}}$ & 10.72 & 10.00 & 1.73 & $\ldots$ & 0.00 & 2006 Nov 23 & 4800 & 0 & $\mathrm{~B}, \mathrm{~S}$ & $3,7,8$ \\
\hline Mrk 477 & $0.03744^{\mathrm{b}}$ & 11.14 & 10.72 & 9.01 & $\ldots$ & 0.00 & 2006 Mar 31 & 3926 & 90 & - & 4 \\
\hline Mrk 506 & $0.04303^{\mathrm{a}}$ & 10.69 & 10.12 & 2.30 & 170 & 2.96 & 2005 Sep 08 & 4830 & 27 & - & 1 \\
\hline Mrk 595 & $0.02739^{\mathrm{b}}$ & 10.64 & 10.28 & 3.31 & 444 & 1.41 & 2006 Nov 23 & 6000 & 0 & S & 3,8 \\
\hline Mrk 1018 & 0.04263 & $\ldots$ & $\ldots$ & $\ldots$ & 414 & 1.58 & 2006 Nov 23 & 6000 & 0 & - & 1 \\
\hline Mrk 1126 & 0.01057 & $\ldots$ & $\cdots$ & $\cdots$ & 540 & 1.70 & 2006 Nov 22 & 7200 & 0 & S & 1,8 \\
\hline NGC 788 & 0.01350 & 10.04 & 9.38 & 0.41 & 226 & 3.20 & 2005 Sep 04 & 2700 & 0 & S & 1,6 \\
\hline NGC 1019 & $0.02460^{\mathrm{a}}$ & 10.43 & 9.97 & 1.62 & 178 & 2.63 & 2006 Nov 22 & 6000 & 0 & $\mathrm{~B}, \mathrm{R}, \mathrm{S}$ & $2,7,8$ \\
\hline NGC 7603 & 0.02956 & 10.78 & 10.41 & 4.43 & 313 & 0.45 & 2006 Nov 23 & 7200 & 0 & $\mathrm{R}, \mathrm{S}$ & $1,4,8$ \\
\hline
\end{tabular}


Table 1 - Continued

\begin{tabular}{cccccccccccc}
\hline \hline $\begin{array}{c}\text { Name } \\
(1)\end{array}$ & $z$ & $\begin{array}{c}L_{\mathrm{IR}} \\
(2)\end{array}$ & $\begin{array}{c}L_{\mathrm{FIR}} \\
(3)\end{array}$ & $\begin{array}{c}\mathrm{SFR} \\
(4)\end{array}$ & $\begin{array}{c}v_{c} \\
(6)\end{array}$ & $\begin{array}{c}W_{\text {eq }} \\
(7)\end{array}$ & $\begin{array}{c}\text { Run } \\
(8)\end{array}$ & $\begin{array}{c}t_{\text {exp }} \\
(9)\end{array}$ & $\begin{array}{c}\text { PA } \\
(10)\end{array}$ & $\begin{array}{c}\text { Structure } \\
(11)\end{array}$ & $\begin{array}{c}\text { Refs } \\
(12)\end{array}$ \\
\hline UGC 3223 & 0.01567 & 10.37 & 10.09 & 2.13 & 263 & 1.66 & 2006 Nov 22 & 6000 & 0 & $\mathrm{~S}$ & $1,4,8$ \\
\hline
\end{tabular}

Note. - Col.(2): Heliocentric redshift. All redshifts are based on stellar measurements except (a) HI 21-cm measurements, or (b) measured from the emission lines in our data. Col.(3): Infrared luminosity, in logarithmic units of $L_{\odot}$. Col.(4): Far-infrared luminosity, in logarithmic units of $L_{\odot}$ (see Section 2.2). Col.(5): Star formation rate, computed from the far-infrared luminosity (Section 2.2). Col.(6): Circular velocity, equal to $\sqrt{2} \sigma$, $v_{r o t}$, or the quadratic combination if both are available. Col.(7): Rest-frame equivalent width of Na I D as computed from our model fits. Col.(8): Observing dates (Section 3). Instrument used was the R-C Spectrograph on the KPNO 4m. Col.(9): Total exposure time in seconds. Col.(10): Slit position angle. Col.(11): Letters indicate dust structure around nucleus (see Section 7.2.2): (B) nuclear dust bar, (F) dust filaments, (I) irregular dust, (R) nuclear dust ring, (S) nuclear dust spiral. Col.(12): Reference.

References. - (1) Nelson \& Whittle 1995; (2) Springob et al. 2005; (3) Whittle 1992a; (4) IRAS Faint Source Catalog; (5) IRAS Point Source Catalog; (6) Martini et al. 2003a; (7) Malkan et al. 1998; (8) Deo et al. 2006. 
Table 2. Properties of individual velocity components

\begin{tabular}{|c|c|c|c|c|c|c|c|}
\hline $\begin{array}{c}\text { Name } \\
(1)\end{array}$ & $\begin{array}{l}\lambda_{1, c} \\
(\AA) \\
(2)\end{array}$ & $\begin{array}{c}\Delta v \\
\left(\mathrm{~km} \mathrm{~s}^{-1}\right) \\
(3)\end{array}$ & $\begin{array}{c}b \\
\left(\mathrm{~km} \mathrm{~s}^{-1}\right) \\
(4)\end{array}$ & $\begin{array}{l}\tau_{1, c} \\
(5)\end{array}$ & (6) & $\begin{array}{c}N(\mathrm{Na} \mathrm{I}) \\
\left(\mathrm{cm}^{-2}\right) \\
(7)\end{array}$ & $\begin{array}{c}N(\mathrm{H}) \\
\left(\mathrm{cm}^{-2}\right) \\
(8)\end{array}$ \\
\hline \multicolumn{8}{|c|}{ Seyfert 2s } \\
\hline Akn 79 & 5999.47 & $-44 \pm 5$ & $85 \pm 19$ & $1.07_{-0.21}^{+0.50}$ & $0.12_{-0.02}^{+0.06}$ & $13.44_{-0.20}^{+0.28}$ & $21.08_{-0.43}^{+0.43}$ \\
\hline$\ldots$ & 5999.86 & $-25 \pm 7$ & $280 \pm 22$ & $0.27_{-0.04}^{+0.04}$ & $0.48_{-0.06}^{+0.07}$ & $13.42{ }_{-0.12}^{+0.12}$ & $21.06_{-0.18}^{+0.18}$ \\
\hline Arp 107A & 6102.90 & $54 \pm 24$ & $323 \pm 59$ & $0.07_{-0.01}^{+0.05}$ & $0.92_{-0.01}^{+0.04}$ & $12.94_{-0.09}^{+0.25}$ & $20.58_{-0.09}^{+0.25}$ \\
\hline Mrk 348 & 5988.26 & $65 \pm 4$ & $261 \pm 25$ & $0.06_{-0.01}^{+0.01}$ & $1.00_{-0.01}^{+0.01}$ & $12.74_{-0.06}^{+0.08}$ & $20.38_{-0.06}^{+0.08}$ \\
\hline Mrk 622 & 6033.19 & $-138 \pm 10$ & $416 \pm 62$ & $0.09_{-0.01}^{+0.04}$ & $0.55_{-0.02}^{+0.23}$ & $13.13_{-0.07}^{+0.16}$ & $20.77_{-0.07}^{+0.16}$ \\
\hline Mrk 686 & 5981.63 & $16 \pm 5$ & $287 \pm 34$ & $0.10_{-0.01}^{+0.04}$ & $1.00_{-0.01}^{+0.01}$ & $13.05_{-0.11}^{+0.25}$ & $20.69_{-0.17}^{+0.31}$ \\
\hline Mrk 1157 & 5986.67 & $3 \pm 2$ & $211 \pm 20$ & $0.83_{-0.13}^{+0.17}$ & $0.19_{-0.03}^{+0.04}$ & $13.79_{-0.13}^{+0.17}$ & $21.43_{-0.20}^{+0.23}$ \\
\hline NGC 1358 & 5977.92 & $35 \pm 6$ & $320 \pm 19$ & $0.43_{-0.05}^{+0.05}$ & $0.37_{-0.04}^{+0.04}$ & $13.69_{-0.08}^{+0.08}$ & $21.33_{-0.15}^{+0.15}$ \\
\hline NGC 1667 & 5986.79 & $-12 \pm 84$ & $343 \pm 19$ & $0.32_{-0.03}^{+0.03}$ & $0.46_{-0.05}^{+0.05}$ & $13.59_{-0.06}^{+0.06}$ & $21.23_{-0.13}^{+0.13}$ \\
\hline NGC 3362 & 6062.43 & $84 \pm 38$ & $323 \pm 92$ & $0.05_{-0.01}^{+0.07}$ & $1.00_{-0.01}^{+0.01}$ & $12.83_{-0.16}^{+0.39}$ & $20.47_{-0.16}^{+0.39}$ \\
\hline NGC 3786 & 5947.88 & $-148 \pm 5$ & $34 \pm 3$ & $5.00_{-2.00}^{+0.36}$ & $0.10_{-0.04}^{+0.01}$ & $>13.76_{-0.23}^{+0.05}$ & $>21.40_{-0.23}^{+0.05}$ \\
\hline$\ldots$ & 5951.19 & $19 \pm 2$ & $239 \pm 14$ & $0.34_{-0.03}^{+0.04}$ & $0.44_{-0.04}^{+0.04}$ & $>13.45_{-0.21}^{+0.03}$ & $>21.09_{-0.21}^{+0.03}$ \\
\hline NGC 4388 & 5948.05 & $51 \pm 17$ & $125 \pm 5$ & $0.98_{-0.07}^{+0.07}$ & $0.32_{-0.02}^{+0.02}$ & $13.60_{-0.04}^{+0.04}$ & $21.24_{-0.04}^{+0.04}$ \\
\hline NGC 5252 & 6034.47 & $39 \pm 10$ & $375 \pm 23$ & $0.10_{-0.01}^{+0.02}$ & $1.00_{-0.01}^{+0.01}$ & $13.14_{-0.07}^{+0.15}$ & $20.78_{-0.13}^{+0.21}$ \\
\hline NGC 5728 & 5952.30 & $-11 \pm 1$ & $262 \pm 26$ & $0.27_{-0.03}^{+0.06}$ & $0.37_{-0.04}^{+0.08}$ & $13.40_{-0.13}^{+0.17}$ & $21.04_{-0.19}^{+0.23}$ \\
\hline NGC 7319 & 6026.74 & $-133 \pm 19$ & $100 \pm 1$ & $3.00_{-2.12}^{+0.24}$ & $0.20_{-0.14}^{+0.02}$ & $13.34_{-0.10}^{+0.04}$ & $20.98_{-0.10}^{+0.04}$ \\
\hline$\ldots$ & 6026.82 & $-130 \pm 1$ & $450 \pm 1$ & $0.46_{-0.06}^{+0.06}$ & $0.49_{-0.06}^{+0.06}$ & $13.81_{-0.12}^{+0.05}$ & $21.45_{-0.12}^{+0.05}$ \\
\hline NGC 7672 & 5976.55 & $-25 \pm 24$ & $98 \pm 6$ & $1.12_{-0.07}^{+0.17}$ & $0.24_{-0.01}^{+0.04}$ & $13.53_{-0.06}^{+0.11}$ & $21.17_{-0.03}^{+0.18}$ \\
\hline NGC 7682 & 6000.21 & $99 \pm 3$ & $228 \pm 21$ & $0.49_{-0.08}^{+0.09}$ & $0.25_{-0.04}^{+0.05}$ & $13.59_{-0.09}^{+0.08}$ & $21.23_{-0.09}^{+0.08}$ \\
\hline UGC 3995 & 5992.99 & $127 \pm 2$ & $268 \pm 15$ & $0.13_{-0.01}^{+0.02}$ & $1.00_{-0.01}^{+0.01}$ & $13.09_{-0.03}^{+0.07}$ & $20.73_{-0.03}^{+0.07}$ \\
\hline \multicolumn{8}{|c|}{ Seyfert 1s } \\
\hline Akn 202 & 6069.22 & $113 \pm 11$ & $129 \pm 32$ & $1.02_{-0.05}^{+0.23}$ & $0.05_{-0.01}^{+0.01}$ & $13.67_{-0.13}^{+0.12}$ & $21.31_{-0.13}^{+0.12}$ \\
\hline Mrk 6 & 6008.03 & $-229 \pm 1$ & $25 \pm 1$ & $1.46_{-0.03}^{+0.03}$ & $0.48_{-0.01}^{+0.01}$ & $13.12_{-0.29}^{+0.17}$ & $20.76_{-0.29}^{+0.17}$ \\
\hline$\ldots$ & 5992.12 & $-1024 \pm 1$ & $16 \pm 11$ & $4.75_{-0.89}^{+0.23}$ & $0.06_{-0.01}^{+0.01}$ & $13.44_{-0.31}^{+0.29}$ & $21.08_{-0.31}^{+0.20}$ \\
\hline Mrk 10 & 6070.59 & $26 \pm 7$ & $305 \pm 34$ & $0.17_{-0.02}^{+0.06}$ & $0.45_{-0.04}^{+0.015}$ & $13.28_{-0.11}^{+0.23}$ & $20.92_{-0.17}^{+0.30}$ \\
\hline Mrk 79 & 6028.25 & $-15 \pm 7$ & $112 \pm 23$ & $0.92_{-0.31}^{+0.32}$ & $0.06_{-0.02}^{+0.06}$ & $13.57_{-0.24}^{+0.11}$ & $21.21_{-0.30}^{+0.31}$ \\
\hline Mrk 352 & 5986.09 & $45 \pm 8$ & $111 \pm 25$ & $0.16_{-0.01}^{+0.04}$ & $0.16_{-0.01}^{+0.03}$ & $12.82_{-0.42}^{+0.07}$ & $20.46_{-0.42}^{+0.07}$ \\
\hline Mrk 359 & 5997.12 & $-17 \pm 11$ & $147 \pm 28$ & $0.21_{-0.03}^{+0.12}$ & $0.20_{-0.03}^{+0.12}$ & $13.03_{-0.17}^{+0.30}$ & $20.67_{-0.23}^{+0.36}$ \\
\hline Mrk 506 & 6154.09 & $135 \pm 7$ & $296 \pm 32$ & $0.17_{-0.01}^{+0.05}$ & $0.64_{-0.05}^{+0.18}$ & $13.26_{-0.06}^{+0.12}$ & $20.90_{-0.06}^{+0.12}$ \\
\hline Mrk 595 & 6059.26 & $8 \pm 5$ & $230 \pm 21$ & $0.15_{-0.01}^{+0.03}$ & $0.44_{-0.03}^{+0.08}$ & $13.09_{-0.05}^{+0.15}$ & $20.73_{-0.12}^{+0.22}$ \\
\hline Mrk 1018 & 6150.34 & $67 \pm 5$ & $379 \pm 31$ & $0.12_{-0.01}^{+0.03}$ & $0.36_{-0.03}^{+0.09}$ & $13.22_{-0.05}^{+0.10}$ & $20.86_{-0.05}^{+0.10}$ \\
\hline Mrk 1126 & 5962.64 & $138 \pm 3$ & $204 \pm 11$ & $0.08_{-0.01}^{+0.01}$ & $1.00_{-0.01}^{+0.01}$ & $12.79_{-0.03}^{+0.06}$ & $20.43_{-0.03}^{+0.06}$ \\
\hline NGC 788 & 5977.35 & $0 \pm 3$ & $253 \pm 61$ & $0.72_{-0.08}^{+0.06}$ & $0.28_{-0.03}^{+0.01}$ & $13.81_{-0.19}^{+0.19}$ & $21.45_{-0.25}^{+0.25}$ \\
\hline NGC 1019 & 6044.02 & $68 \pm 4$ & $253 \pm 23$ & $0.31+0.05$ & $0.41_{-0.05}^{+0.07}$ & $13.44_{-0.07}^{+0.07}$ & $21.08_{-0.07}^{+0.07}$ \\
\hline
\end{tabular}


Table 2 - Continued

\begin{tabular}{|c|c|c|c|c|c|c|c|}
\hline $\begin{array}{c}\text { Name } \\
(1)\end{array}$ & $\begin{array}{l}\lambda_{1, c} \\
(\AA) \\
(2)\end{array}$ & $\begin{array}{c}\Delta v \\
\left(\mathrm{~km} \mathrm{~s}^{-1}\right) \\
(3)\end{array}$ & $\begin{array}{c}b \\
\left(\mathrm{~km} \mathrm{~s}^{-1}\right) \\
(4)\end{array}$ & $\begin{array}{l}\tau_{1, c} \\
(5)\end{array}$ & $\begin{array}{l}C_{f} \\
(6)\end{array}$ & $\begin{array}{c}N(\mathrm{Na} \mathrm{I}) \\
\left(\mathrm{cm}^{-2}\right) \\
(7)\end{array}$ & $\begin{array}{c}N(\mathrm{H}) \\
\left(\mathrm{cm}^{-2}\right) \\
(8)\end{array}$ \\
\hline NGC 7603 & 6073.78 & $93 \pm 11$ & $497 \pm 45$ & $0.06_{-0.05}^{+0.04}$ & $0.14_{-0.11}^{+0.10}$ & $13.06_{-0.70}^{+0.22}$ & $20.70_{-0.70}^{+0.22}$ \\
\hline UGC 3223 & 5989.19 & $-39 \pm 1$ & $81 \pm 10$ & $5.00_{-1.89}^{+0.77}$ & $0.08_{-0.03}^{+0.01}$ & $14.16_{-0.17}^{+0.17}$ & $21.80_{-0.17}^{+0.17}$ \\
\hline 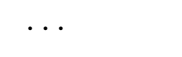 & 5991.73 & $88 \pm 1$ & $98 \pm 22$ & $0.92_{-0.20}^{+0.39}$ & $0.16_{-0.04}^{+0.07}$ & $13.50_{-0.13}^{+0.12}$ & $21.14_{-0.13}^{+0.12}$ \\
\hline
\end{tabular}

Note. - Col.(2): Redshifted heliocentric wavelength, in vacuum, of the Na I $\mathrm{D}_{1} \lambda 5896$ line. Col.(3): Velocity relative to systemic. Negative velocities are blueshifted, positive are redshifted. Components with $\Delta v<-50 \mathrm{~km} \mathrm{~s}^{-1}$ and $|\Delta v|>2 \delta(\Delta v)$ are assumed to be outflowing; those with $\Delta v>50 \mathrm{~km} \mathrm{~s}^{-1}$ and $|\Delta v|>2 \delta(\Delta v)$ are assumed to be inflowing . Col.(4): Doppler parameter. Col. (5): Central optical depth of the $\mathrm{Na} I \mathrm{D}_{1} \lambda 5896$ line; the optical depth of the $\mathrm{D}_{2}$ line is twice this value. Col.(6): Covering fraction of the gas. Col.(7-8): Logarithm of column density of $\mathrm{Na} \mathrm{I}$ and $\mathrm{H}$, respectively.

Table 3. Outflow: Individual objects

\begin{tabular}{lccccccc}
\hline \hline $\begin{array}{c}\text { Name } \\
(1)\end{array}$ & $\begin{array}{c}\Delta v_{\max } \\
\left(\mathrm{km} \mathrm{s}^{-1}\right) \\
(2)\end{array}$ & $\begin{array}{c}M \\
\left(M_{\odot}\right) \\
(3)\end{array}$ & $\begin{array}{c}d M / d t \\
\left(\mathrm{M}_{\odot} \mathrm{yr}^{-1}\right) \\
(4)\end{array}$ & $\begin{array}{c}p \\
(\text { dyn s }) \\
(5)\end{array}$ & $\begin{array}{c}d p / d t \\
(\mathrm{dyn}) \\
(6)\end{array}$ & $\begin{array}{c}E \\
(\mathrm{ergs}) \\
(7)\end{array}$ & $\begin{array}{c}d E / d t \\
\left(\mathrm{ergs} \mathrm{s}^{-1}\right) \\
(8)\end{array}$ \\
\hline \multicolumn{7}{c}{ Seyfert 2s } \\
\hline Mrk 622 & -484 & 8.76 & 1.22 & 49.2 & 34.2 & 56.1 & 41.0 \\
NGC 3786 & -176 & 8.64 & 1.12 & 49.1 & 34.1 & 56.0 & 41.0 \\
NGC 7319 & -217 & 8.52 & 0.95 & 48.9 & 33.9 & 55.8 & 40.7 \\
$\ldots$ & -504 & 9.39 & 1.85 & 49.8 & 34.8 & 56.7 & 41.7 \\
\hline \multicolumn{7}{c}{ Seyfert 1s } \\
\hline Mrk 6 & -250 & 8.69 & 1.36 & 49.4 & 34.5 & 56.4 & 41.6 \\
$\ldots$ & -1037 & 8.08 & 1.40 & 49.4 & 35.2 & 57.1 & 42.9 \\
\hline
\end{tabular}

Note. - Col.(2): Maximum velocity in the outflow, $\Delta v_{\max } \equiv \Delta v-\mathrm{FWHM} / 2$. Col .(3): Log of total outflowing mass. Col.(4): Log of mass outflow rate. Col. (5): Log of total momentum of outflow. Col.(6): Log of momentum outflow rate. Col.(7): Log of total kinetic energy of outflow. Col.(8): Log of kinetic energy outflow rate. Note that all values are calculated using $\Omega \sim 0.1$. 
Table 4. Outflow: Average properties

\begin{tabular}{|c|c|c|c|c|}
\hline $\begin{array}{l}\text { Quantity } \\
\text { (1) }\end{array}$ & $\begin{array}{c}\text { IR-Faint Seyfert } 2 \mathrm{~s} \\
(2)\end{array}$ & $\begin{array}{l}\text { IR-Faint Seyfert 1s } \\
(3)\end{array}$ & $\begin{array}{c}\text { IR-Lum. Seyfert } 2 \mathrm{~s} \\
(4)\end{array}$ & $\begin{array}{c}\text { IR-Lum. Seyfert } 1 \mathrm{~s} \\
(5)\end{array}$ \\
\hline Number of galaxies & 17 & 18 & 20 & 6 \\
\hline Detection rate (\%) & $18 \pm 9$ & $6 \pm 6$ & $45 \pm 11$ & $50 \pm 20$ \\
\hline \multicolumn{5}{|c|}{ Galaxy Properties } \\
\hline 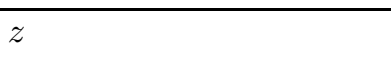 & $0.018 \pm 0.01$ & $0.025 \pm 0.01$ & $0.148 \pm 0.11$ & $0.150 \pm 0.09$ \\
\hline $\log \left(L_{\mathrm{FIR}} / L_{\odot}\right)$ & $10.02 \pm 0.35$ & $10.14 \pm 0.23$ & $11.86 \pm 0.31$ & $12.13 \pm 0.1$ \\
\hline $\operatorname{SFR}\left(\mathrm{M}_{\odot} \mathrm{yr}^{-1}\right)$ & $2.39 \pm 1.92$ & $2.99 \pm 2.13$ & $118 \pm 109$ & $164 \pm 75$ \\
\hline$\Delta v\left(\mathrm{~km} \mathrm{~s}^{-1}\right)$ & $-137 \pm 8$ & $-627 \pm 562$ & $-322 \pm 388$ & $-4942 \pm 2831$ \\
\hline$\Delta v_{\max }\left(\mathrm{km} \mathrm{s}^{-1}\right)$ & $-345 \pm 173$ & $-643 \pm 556$ & $-618 \pm 422$ & $-5210 \pm 4306$ \\
\hline $\log \left[N(\mathrm{Na} \mathrm{I} \mathrm{D}) / \mathrm{cm} \mathrm{s}^{-2}\right]$ & $13.51 \pm 0.33$ & $13.28 \pm 0.23$ & $13.5 \pm 0.7$ & $14.5 \pm 0.8$ \\
\hline $\log \left[N(\mathrm{H}) / \mathrm{cm} \mathrm{s}^{-2}\right]$ & $21.15 \pm 0.33$ & $20.92 \pm 0.23$ & $20.9 \pm 0.7$ & $21.8 \pm 0.8$ \\
\hline \multicolumn{5}{|c|}{ Velocity Component Properties } \\
\hline$\tau$ & $0.76_{-0.7}^{+0.1}$ & $1.01_{-0.1}^{+0.5}$ & $0.27_{-0.2}^{+0.7}$ & $0.69_{-0.5}^{+1.6}$ \\
\hline$b\left(\mathrm{~km} \mathrm{~s}^{-1}\right)$ & $250 \pm 214$ & $21 \pm 6$ & $232 \pm 182$ & $87 \pm 130$ \\
\hline$C_{f}$ & $0.34_{-0.1}^{+0.1}$ & $0.27_{-0.1}^{+0.1}$ & $0.42_{-0.2}^{+0.5}$ & $0.67_{-0.4}^{+1.2}$ \\
\hline
\end{tabular}

Note. - "IR-Lum." refers to the IR-luminous data of RVS05c. For most quantities we list the mean and $1 \sigma$ dispersions, under the assumption of a Gaussian distribution in the log of the quantity. Statistics for all quantities except $z, L_{\mathrm{FIR}}$, and SFR are computed only for outflowing velocity components. Note that the entries under IR-Faint Seyfert 2s (1s) corresponds to only three (one) objects. 
Table 5. Inflow: Individual objects

\begin{tabular}{|c|c|c|c|c|c|c|c|}
\hline $\begin{array}{c}\text { Name } \\
(1)\end{array}$ & $\begin{array}{c}\Delta v_{\max } \\
\left(\mathrm{km} \mathrm{s}^{-1}\right) \\
(2)\end{array}$ & $\begin{array}{c}M \\
\left(M_{\odot}\right) \\
(3)\end{array}$ & $\begin{array}{c}d M / d t \\
\left(\mathrm{M}_{\odot} \mathrm{yr}^{-1}\right) \\
(4)\end{array}$ & $\begin{array}{c}p \\
\text { (dyn s) } \\
(5)\end{array}$ & $\begin{array}{c}d p / d t \\
(\mathrm{dyn}) \\
(6)\end{array}$ & $\begin{array}{c}E \\
(\mathrm{ergs}) \\
(7)\end{array}$ & $\begin{array}{c}d E / d t \\
\left(\operatorname{ergs~s}^{-1}\right) \\
(8)\end{array}$ \\
\hline \multicolumn{8}{|c|}{ Seyfert 2s } \\
\hline Arp 107A & 323 & 7.39 & 0.15 & 48.4 & 32.7 & 53.9 & 39.2 \\
\hline Mrk 348 & 282 & 7.22 & 0.04 & 47.3 & 32.7 & 53.8 & 39.2 \\
\hline NGC 3362 & 352 & 7.32 & 0.27 & 47.6 & 33.0 & 54.2 & 39.6 \\
\hline NGC 4388 & 155 & 7.59 & 0.31 & 47.6 & 32.8 & 54.0 & 39.2 \\
\hline NGC 7682 & 289 & 7.48 & 0.49 & 47.8 & 33.3 & 54.5 & 40.0 \\
\hline UGC 3995 & 350 & 7.58 & 0.69 & 48.0 & 33.6 & 54.8 & 40.4 \\
\hline \multicolumn{8}{|c|}{ Seyfert 1s } \\
\hline Akn 202 & 220 & 6.89 & -0.04 & 47.3 & 32.8 & 54.0 & 39.6 \\
\hline Mrk 506 & 381 & 7.55 & 0.69 & 48.0 & 33.6 & 54.8 & 40.5 \\
\hline Mrk 1018 & 382 & 7.27 & 0.10 & 47.4 & 32.7 & 53.9 & 39.3 \\
\hline Mrk 1126 & 308 & 7.27 & 0.43 & 47.7 & 33.4 & 54.6 & 40.2 \\
\hline NGC 1019 & 279 & 7.54 & 0.39 & 47.7 & 33.0 & 54.2 & 39.6 \\
\hline NGC 7603 & 507 & 6.70 & -0.32 & 47.0 & 32.5 & 53.6 & 39.1 \\
\hline UGC 3223 & 169 & 7.19 & 0.15 & 47.4 & 32.9 & 54.1 & 39.5 \\
\hline
\end{tabular}

Note. - All inflow dynamics calculations assume an absorber radius of $1 \mathrm{kpc}$. Col.(2): Maximum velocity of the inflow, $\Delta v_{\max } \equiv \Delta v+\mathrm{FWHM} / 2$. Col.(3): Log of total inflowing mass. Col.(4): Log of mass inflow rate. Col. (5): Log of total momentum of inflow. Col.(6): Log of momentum inflow rate. Col.(7): Log of total kinetic energy of inflow. Col.(8): Log of kinetic energy inflow rate. 
Table 6. Inflow: Average properties

\begin{tabular}{lcc}
\hline \hline \multicolumn{1}{c}{$\begin{array}{c}\text { Quantity } \\
(1)\end{array}$} & $\begin{array}{c}\text { IR-Faint Seyfert } 2 \mathrm{~s} \\
(2)\end{array}$ & $\begin{array}{c}\text { IR-Faint Seyfert 1s } \\
(3)\end{array}$ \\
\hline Number of galaxies & 17 & 18 \\
Detection rate $(\%)$ & $35 \pm 11$ & $39 \pm 12$ \\
\hline \multicolumn{3}{c}{ Galaxy Properties } \\
\hline$z$ & $0.018 \pm 0.01$ & $0.025 \pm 0.01$ \\
$\log \left(L_{\mathrm{FIR}} / L_{\odot}\right)$ & $10.02 \pm 0.35$ & $10.14 \pm 0.23$ \\
$\mathrm{SFR}\left(\mathrm{M}_{\odot} \mathrm{yr}^{-1}\right)$ & $2.39 \pm 1.92$ & $2.99 \pm 2.13$ \\
$\Delta v\left(\mathrm{~km} \mathrm{~s}^{-1}\right)$ & $80 \pm 29$ & $100 \pm 29$ \\
$\Delta v_{\text {max }}\left(\mathrm{km} \mathrm{s}^{-1}\right)$ & $291 \pm 37$ & $321 \pm 29$ \\
$\log \left[N(\mathrm{Na} \mathrm{I} \mathrm{D}) / \mathrm{cm} \mathrm{s}^{-2}\right]$ & $13.13 \pm 0.38$ & $13.28 \pm 0.29$ \\
$\log \left[N(\mathrm{H}) / \mathrm{cm} \mathrm{s}^{-2}\right]$ & $20.77 \pm 0.38$ & $20.92 \pm 0.29$ \\
\hline \multicolumn{3}{c}{ Velocity Component Properties } \\
\hline$\tau$ & $0.3_{-0.06}^{+0.13}$ & $0.38_{-0.03}^{+0.05}$ \\
$b\left(\mathrm{~km} \mathrm{~s}^{-1}\right)$ & $254 \pm 74$ & $265 \pm 140$ \\
$C_{f}$ & $0.75_{-0.05}^{+0.09}$ & $0.39{ }_{-0.02}^{+0.02}$ \\
\hline
\end{tabular}

Note. - For most quantities we list the mean and $1 \sigma$ dispersions, under the assumption of a Gaussian distribution in the log of the quantity. Statistics for all quantities except $z, L_{\mathrm{FIR}}$, and SFR are computed only for inflowing velocity components. 
Table 7. Statistical comparisons of kinematic parameters: IR-faint and IR-luminous Seyfert galaxies

\begin{tabular}{|c|c|c|}
\hline Samples & $P($ null, $\mathrm{K}-\mathrm{S})$ & $P($ null, Kuiper $)$ \\
\hline \multicolumn{3}{|l|}{$\Delta v$} \\
\hline IR-Faint Seyfert 1s vs IR-Faint Seyfert 2s & 0.76 & 0.69 \\
\hline IR-Faint Seyfert 1s vs IR-Luminous Seyfert 1s & $<0.01$ & $<0.01$ \\
\hline IR-Faint Seyfert 2s vs IR-Luminous Seyfert $2 \mathrm{~s}$ & 0.04 & 0.07 \\
\hline IR-Faint Seyferts vs IR-Luminous Seyferts & $<0.01$ & $<\mathbf{0 . 0 1}$ \\
\hline \multicolumn{3}{|l|}{$\Delta v_{\max }$} \\
\hline IR-Faint Seyfert 1s vs IR-Faint Seyfert 2s & 0.84 & 0.95 \\
\hline IR-Faint Seyfert 1s vs IR-Luminous Seyfert 1s & $<0.01$ & $<0.01$ \\
\hline IR-Faint Seyfert $2 \mathrm{~s}$ vs IR-Luminous Seyfert $2 \mathrm{~s}$ & $<0.01$ & 0.03 \\
\hline IR-Faint Seyferts vs IR-Luminous Seyferts & $<0.01$ & $<\mathbf{0 . 0 1}$ \\
\hline \multicolumn{3}{|l|}{ Doppler Parameter } \\
\hline IR-Faint Seyfert 1s vs IR-Faint Seyfert 2s & 0.33 & 0.71 \\
\hline IR-Faint Seyfert 1s vs IR-Luminous Seyfert 1s & 0.09 & 0.06 \\
\hline IR-Faint Seyfert $2 \mathrm{~s}$ vs IR-Luminous Seyfert $2 \mathrm{~s}$ & 0.28 & 0.72 \\
\hline IR-Faint Seyferts vs IR-Luminous Seyferts & 0.23 & 0.41 \\
\hline
\end{tabular}

Note. - $P($ null $)$ is the probability that the two listed distributions are taken from the same parent population. Categories which have $P($ null $)<0.1$ for both tests are printed in bold. Values are based on all absorption features. 
Table 8. Statistical comparisons of kinematic parameters: All galaxies

\begin{tabular}{lrr}
\hline \hline \multicolumn{1}{c}{ Samples } & $P($ null, K-S $)$ & $P($ null, Kuiper $)$ \\
\hline \multicolumn{4}{c}{$\boldsymbol{\Delta}$} & \\
\hline Seyfert 1s vs Starburst ULIRGs \& LIRGs & $<\mathbf{0 . 0 1}$ & $<\mathbf{0 . 0 1}$ \\
Seyfert 2s vs Starburst ULIRGs \& LIRGs & $\mathbf{0 . 0 3}$ & $\mathbf{0 . 0 9}$ \\
Seyfert 1s vs Seyfert 2s & $<\mathbf{0 . 0 1}$ & $<\mathbf{0 . 0 1}$ \\
\hline \multicolumn{4}{c}{$\boldsymbol{\Delta} \boldsymbol{v}_{\mathbf{m a x}}$} \\
\hline Seyfert 1s vs Starburst ULIRGs \& LIRGs & $<\mathbf{0 . 0 1}$ & $<\mathbf{0 . 0 1}$ \\
Seyfert 2s vs Starburst ULIRGs \& LIRGs & $\mathbf{0 . 0 2}$ & $\mathbf{0 . 0 2}$ \\
Seyfert 1s vs Seyfert 2s & $<\mathbf{0 . 0 1}$ & $<\mathbf{0 . 0 1}$ \\
\hline \multicolumn{4}{c}{ Doppler Parameter } \\
\hline Seyfert 1s vs Starburst ULIRGs \& LIRGs & 0.21 & 0.03 \\
Seyfert 2s vs Starburst ULIRGs \& LIRGs & $<\mathbf{0 . 0 1}$ & $<\mathbf{0 . 0 1}$ \\
Seyfert 1s vs Seyfert 2s & $\mathbf{0 . 0 2}$ & $<\mathbf{0 . 0 1}$ \\
\hline
\end{tabular}

Note. $-P($ null $)$ is the probability that the two listed distributions are taken from the same intrinsic distribution. Categories which have $P($ null $)<0.1$ for both tests are printed in bold. Values are based on all absorption features, both inflowing and outflowing. Note that Seyfert 1 and Seyfert 2 galaxies include both IR-Faint Seyferts from the present study, as well as IR-Luminous Seyferts from RVS05c. Starburst data are taken from RVS05b. 
Table 9. Statistical comparisons of kinematic parameters: Galaxies with outflows

\begin{tabular}{lrr}
\hline \hline \multicolumn{1}{c}{ Samples } & $P($ null, K-S $)$ & $P($ null, Kuiper $)$ \\
\hline \multicolumn{4}{c}{$\boldsymbol{\Delta}$} & & \\
\hline Seyfert 1s vs Starburst ULIRGs \& LIRGs & $<\mathbf{0 . 0 1}$ & $<\mathbf{0 . 0 1}$ \\
Seyfert 2s vs Starburst ULIRGs \& LIRGs & 0.96 & 0.84 \\
Seyfert 1s vs Seyfert 2s & $<\mathbf{0 . 0 1}$ & $<\mathbf{0 . 0 1}$ \\
\hline \multicolumn{4}{c}{$\boldsymbol{\Delta} \boldsymbol{v}_{\mathbf{m a x}}$} \\
\hline Seyfert 1s vs Starburst ULIRGs \& LIRGs & $<\mathbf{0 . 0 1}$ & $<\mathbf{0 . 0 1}$ \\
Seyfert 2s vs Starburst ULIRGs \& LIRGs & 0.26 & 0.31 \\
Seyfert 1s vs Seyfert 2s & $<\mathbf{0 . 0 1}$ & $<\mathbf{0 . 0 1}$ \\
\hline \multicolumn{4}{c}{ Doppler Parameter } \\
\hline Seyfert 1s vs Starburst ULIRGs \& LIRGs & $<\mathbf{0 . 0 1}$ & $<\mathbf{0 . 0 1}$ \\
Seyfert 2s vs Starburst ULIRGs \& LIRGs & 0.24 & 0.28 \\
Seyfert 1s vs Seyfert 2s & $\mathbf{0 . 0 2}$ & $\mathbf{0 . 0 1}$ \\
\hline
\end{tabular}

Note. $-P($ null $)$ is the probability that the two listed distributions are taken from the same parent population. Categories which have $P($ null $)<0.1$ for both tests are printed in bold. Values are based on outflowing absorption only. Note that Seyfert 1 and Seyfert 2 galaxies include both IR-Faint Seyferts from the present study, as well as IR-Luminous Seyferts from RVS05c. Starburst data are taken from RVS05b.

Table 10. Statistical comparisons of kinematic parameters: Seyfert 1s versus Seyfert 2s with inflows

\begin{tabular}{lcc}
\hline \hline \multicolumn{1}{c}{ Samples } & $P($ null, K-S $)$ & $P($ null, Kuiper $)$ \\
\hline$\Delta v$ & 0.83 & 0.87 \\
$\Delta v_{\text {max }}$ & 0.72 & 0.89 \\
Doppler Parameter & 0.68 & 0.99 \\
\hline
\end{tabular}

Note. - $P$ (null) is the probability that the two listed distributions are taken from the same parent population. Values are based on objects with inflowing absorption only, and include data for IR-faint galaxies from this survey and IR-bright galaxies from RVS05c. 

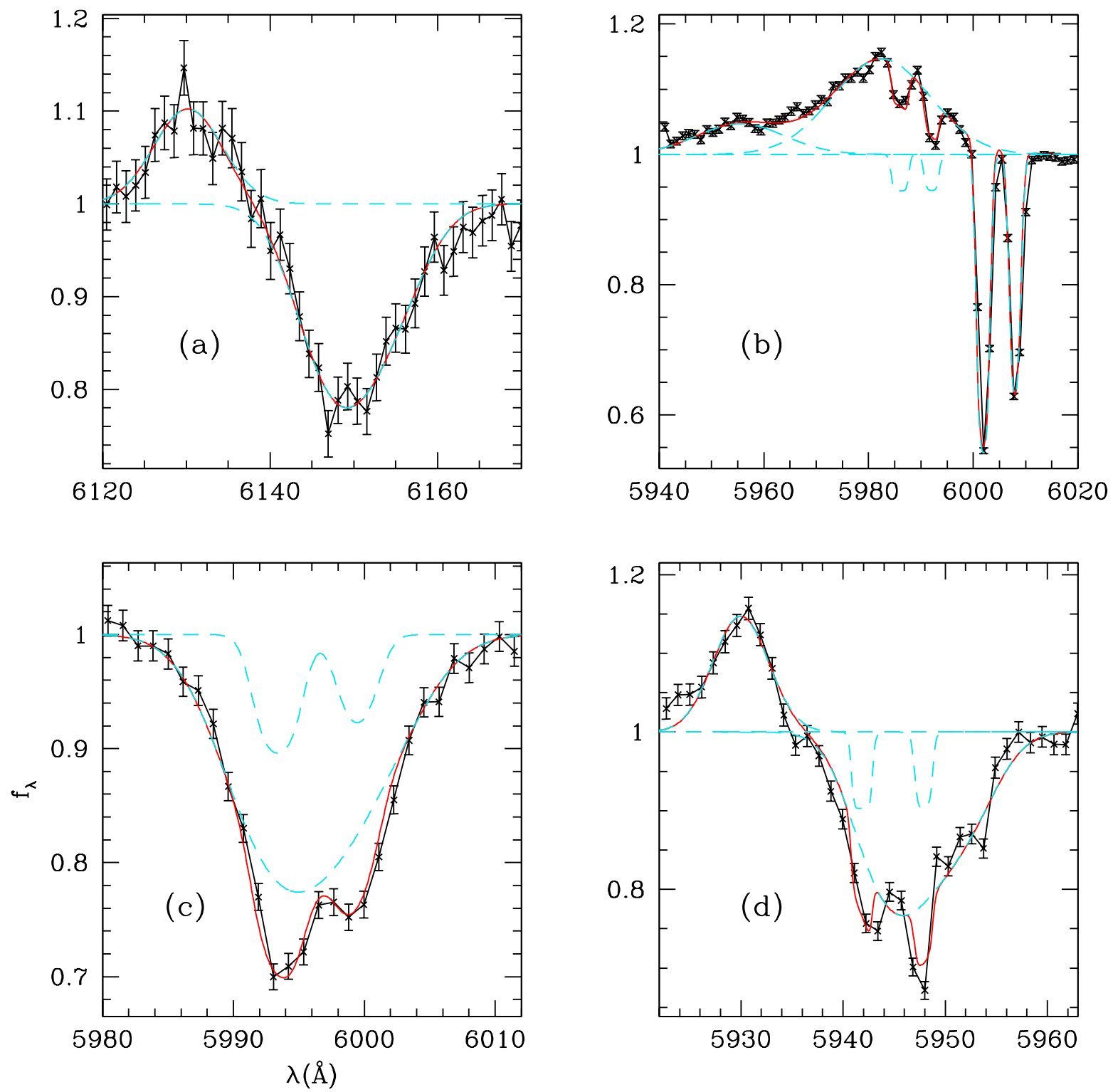

Fig. 1.- Binned data showing the He I and Na I D complexes, plotted on an intensity versus wavelength scale, for four objects. In all cases, blue dashed lines correspond to the individual He I or Na I D line fits, with solid red lines showing the combined He I and Na I D fit for the object. Panels (a) and (b) show two Seyfert 1 galaxies, Mrk 506 and Mrk 6. Mrk 506 is fit with one He I and one Na I D velocity component. Mrk 6 is fit with two He I components and two Na I D velocity components. Panels (c) and (d) show two Seyfert 2 galaxies, Akn 79 and NGC 3786. Akn 79 does not have a resolvable He I line; two Na I D components, one narrow and one broad, are used in the fit. NGC 3786 is fit with one He I component and two $\mathrm{Na}$ I D , one narrow and one broad. Note that the data for NGC 3786 are low signal-to-noise compared to most other objects, and thus the lines are not quite as well constrained. 


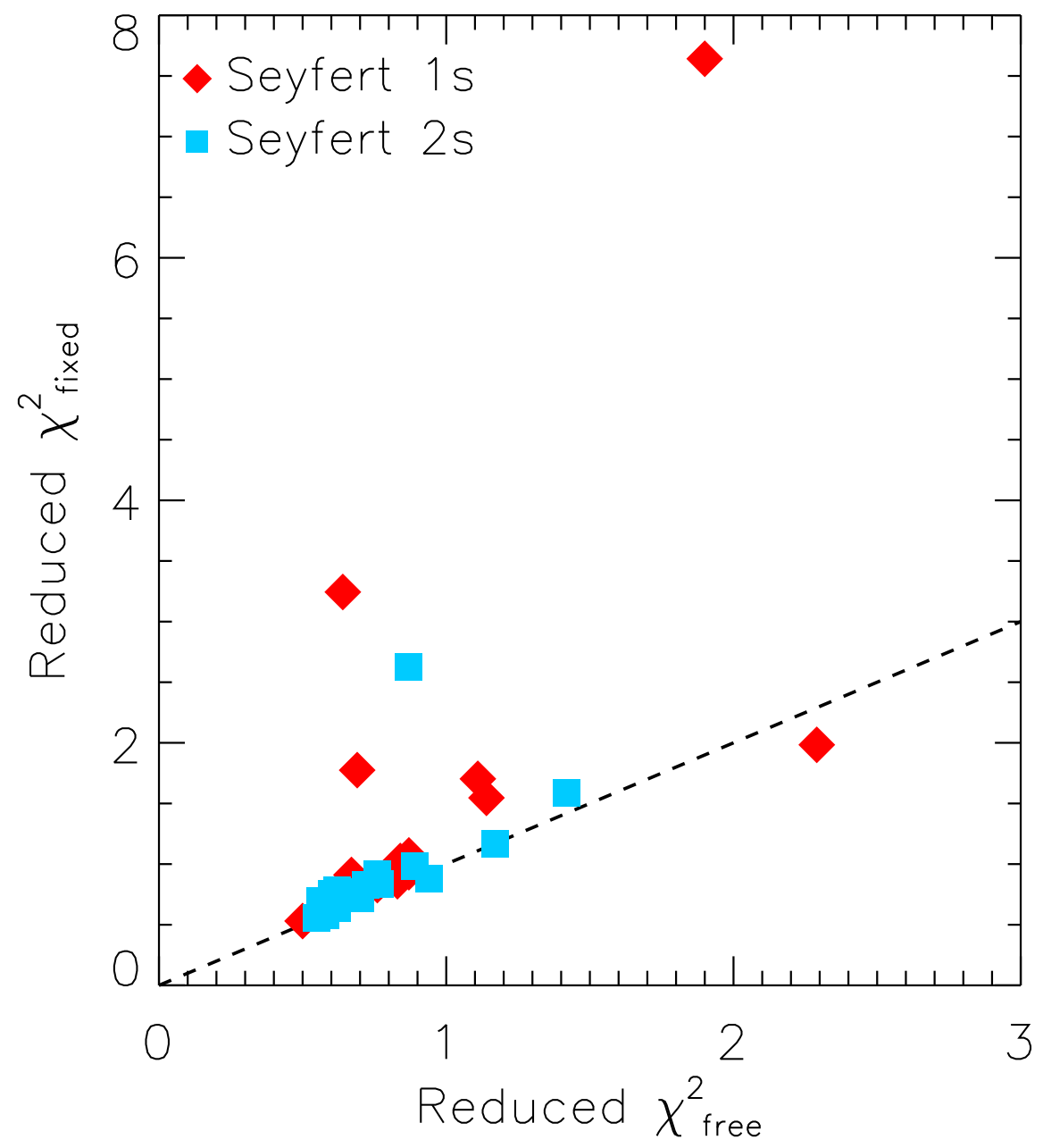

Fig. 2.- Reduced $\chi^{2}$ values for free-floating and $\mathrm{H} \alpha$-fixed He I parameter fits. See Section 4.1 for an explanation of these fits. The dashed black line corresponds to equal $\chi^{2}$ values. The free fits generally give lower $\chi^{2}$. The results from these fits were adopted in the present study. 

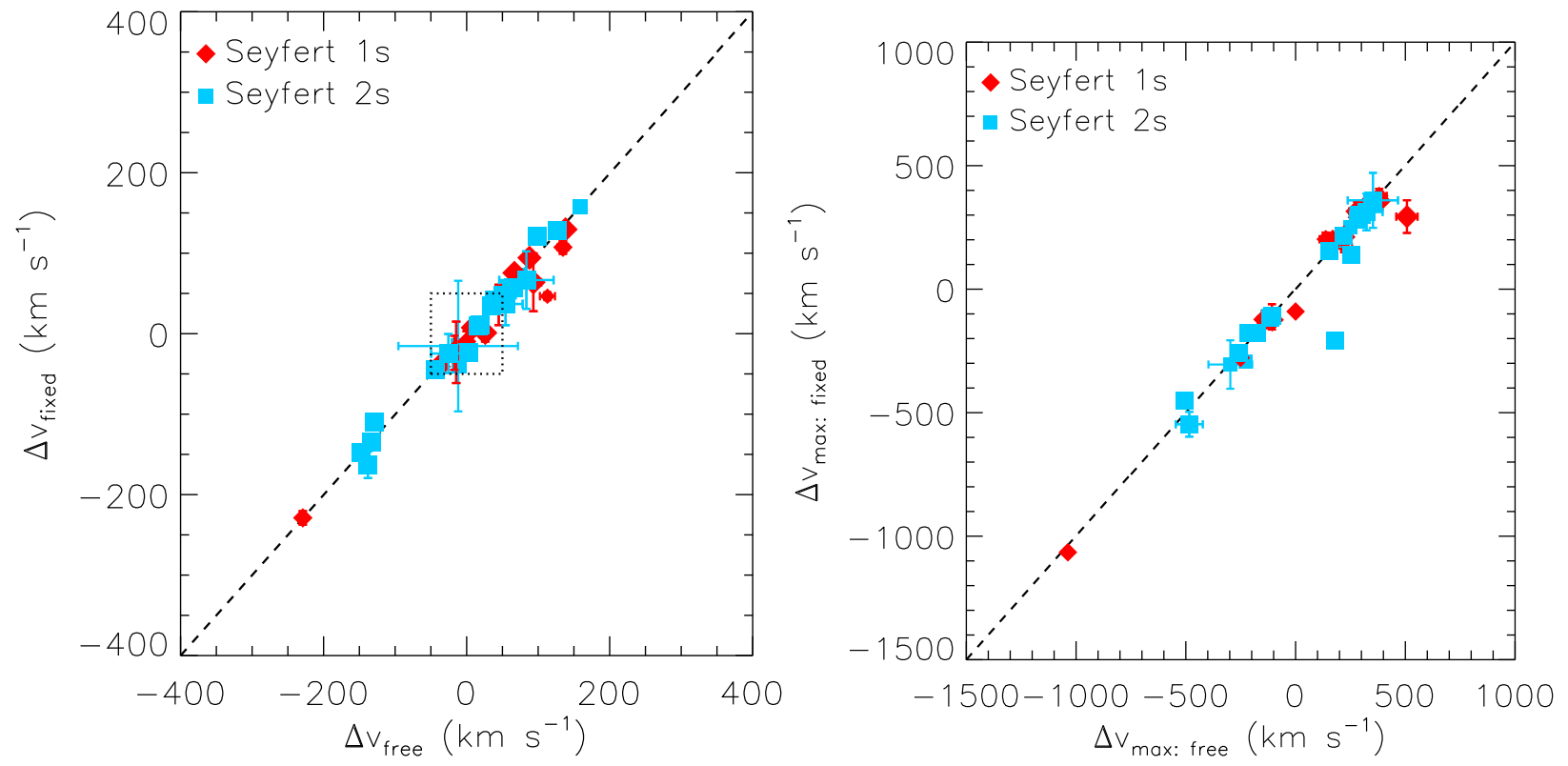

Fig. 3.- Left: $\Delta v$ for $\mathrm{H} \alpha$-fixed He I fit versus $\Delta v$ for free-floating He I fit. Smaller points are objects with larger redshift uncertainty. Dashed black line through center corresponds to equal fixed and free values, and all points outside dashed black box have $|\Delta v|>50 \mathrm{~km} \mathrm{~s}^{-1}$. Right: Same, but for $\Delta v_{\max }$. The two types of fits give very similar results. 


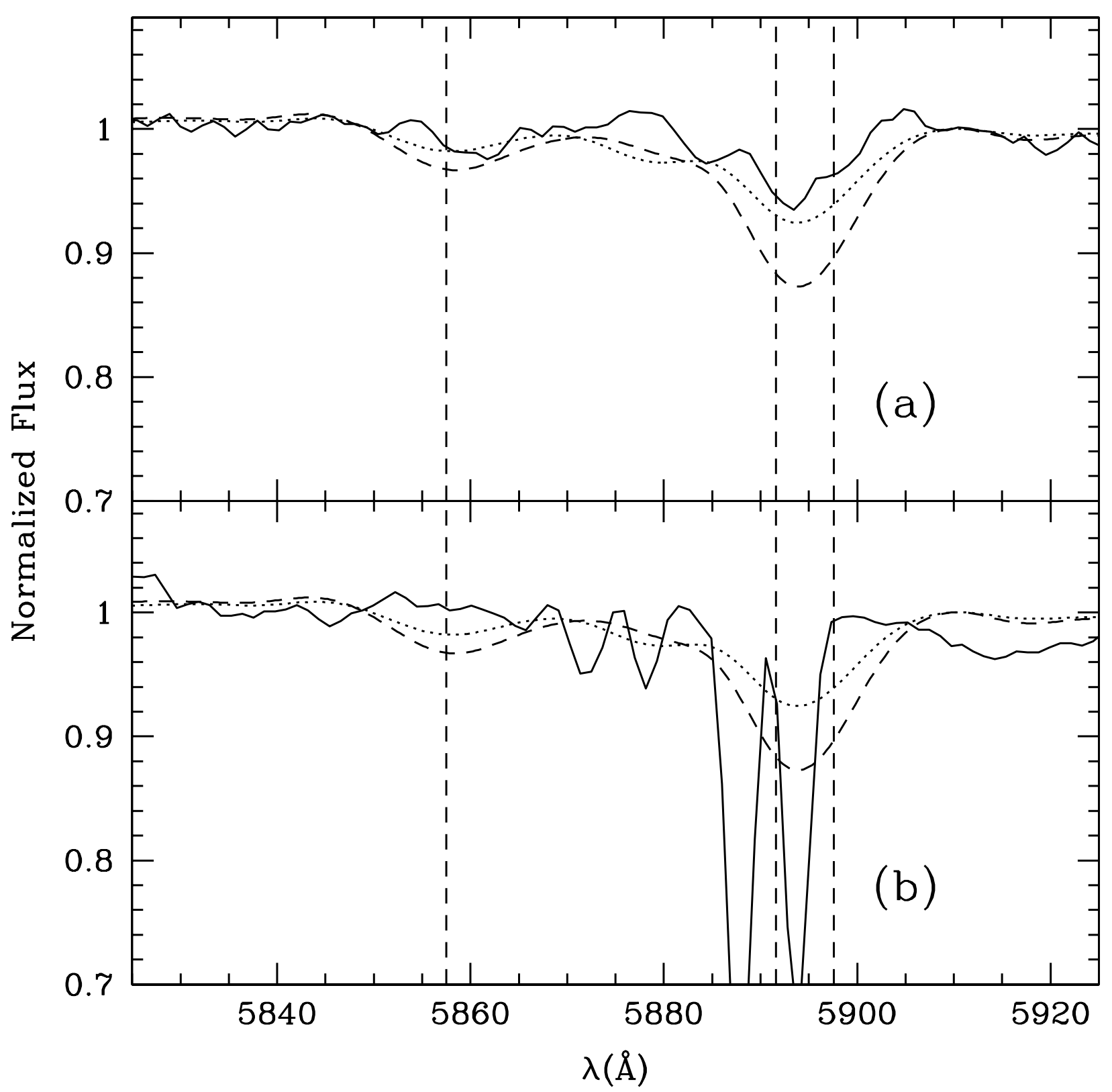

Fig. 4. - Spectra of two Seyfert 1 galaxies, (a) Mrk 352 and (b) Mrk 6, in the Na I D region. These spectra were He I emission line subtracted, boxcar smoothed, and normalized to unity at $5910 \AA$. The dotted line shows the 10/90 model (10\%/90\% stellar mass ratio for a young 40 Myr instantaneous burst population compared to an older 10 Gyr population) and the dashed line shows the 1/99 model (1\%/99\% young to old stellar mass ratio), as outlined in Section 4.4. Strong stellar features are marked with dashed vertical lines. Mrk 352 shows absorption that is weaker than the stellar models, suggesting that the absorption is only stellar or atmospheric (the feature at $\sim 5880 \AA$ was also determined to be atmospheric). In contrast, Mrk 6 shows very strong absorption, which is consistent with predominant interstellar origin. 


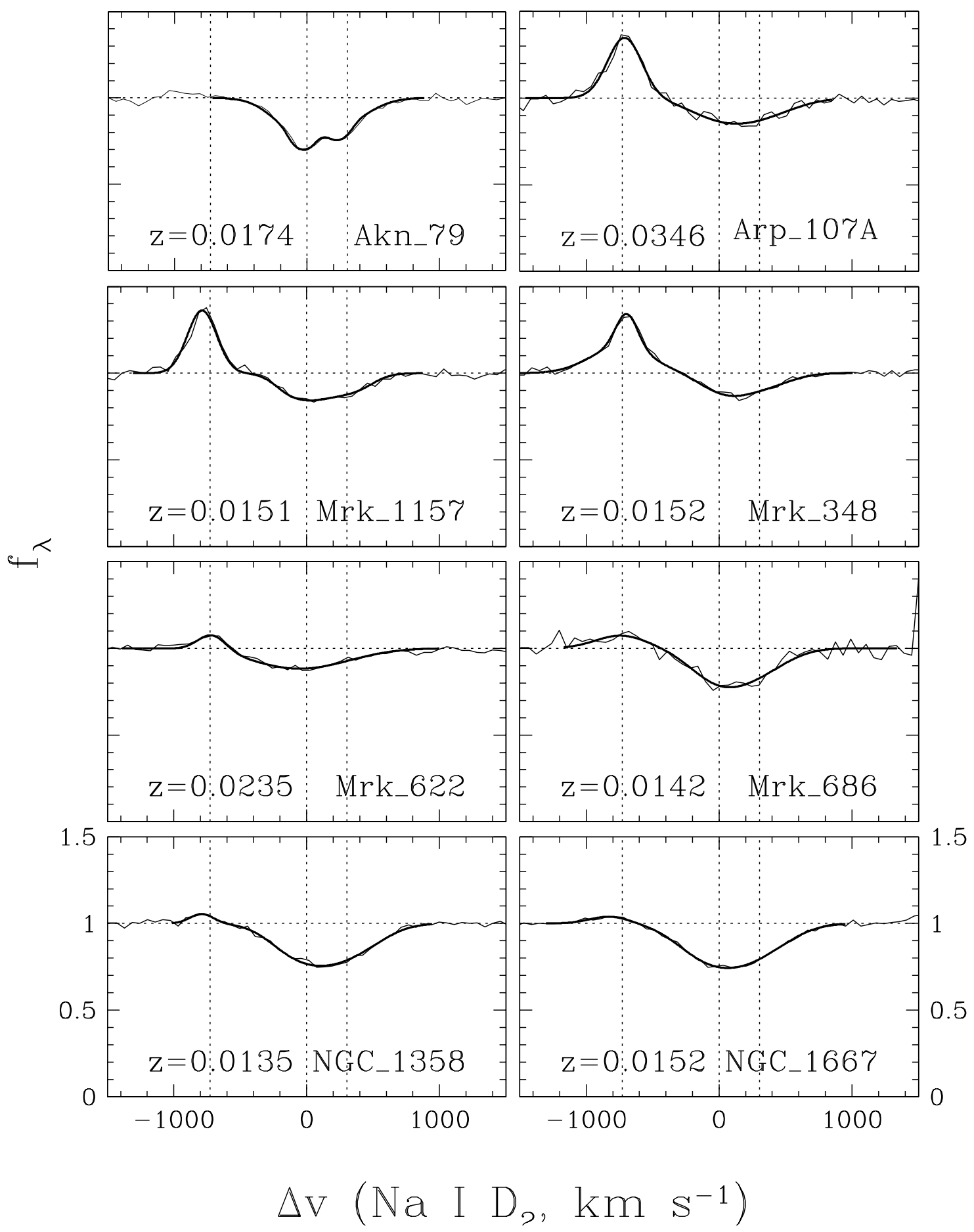

Fig. 5.- Spectra of the He I emission + Na I D absorption region in infrared-faint Seyfert 2 galaxies. These spectra are plotted on a velocity scale based on the systemic Na I D $\lambda 5890$ velocity. The thin lines are smoothed original spectra, and thick lines are fits to the data. The vertical dotted lines show the locations of the Na I D $\lambda \lambda 5890,5896$ doublet and He I $\lambda 5876$ emission line in the object's rest frame. 


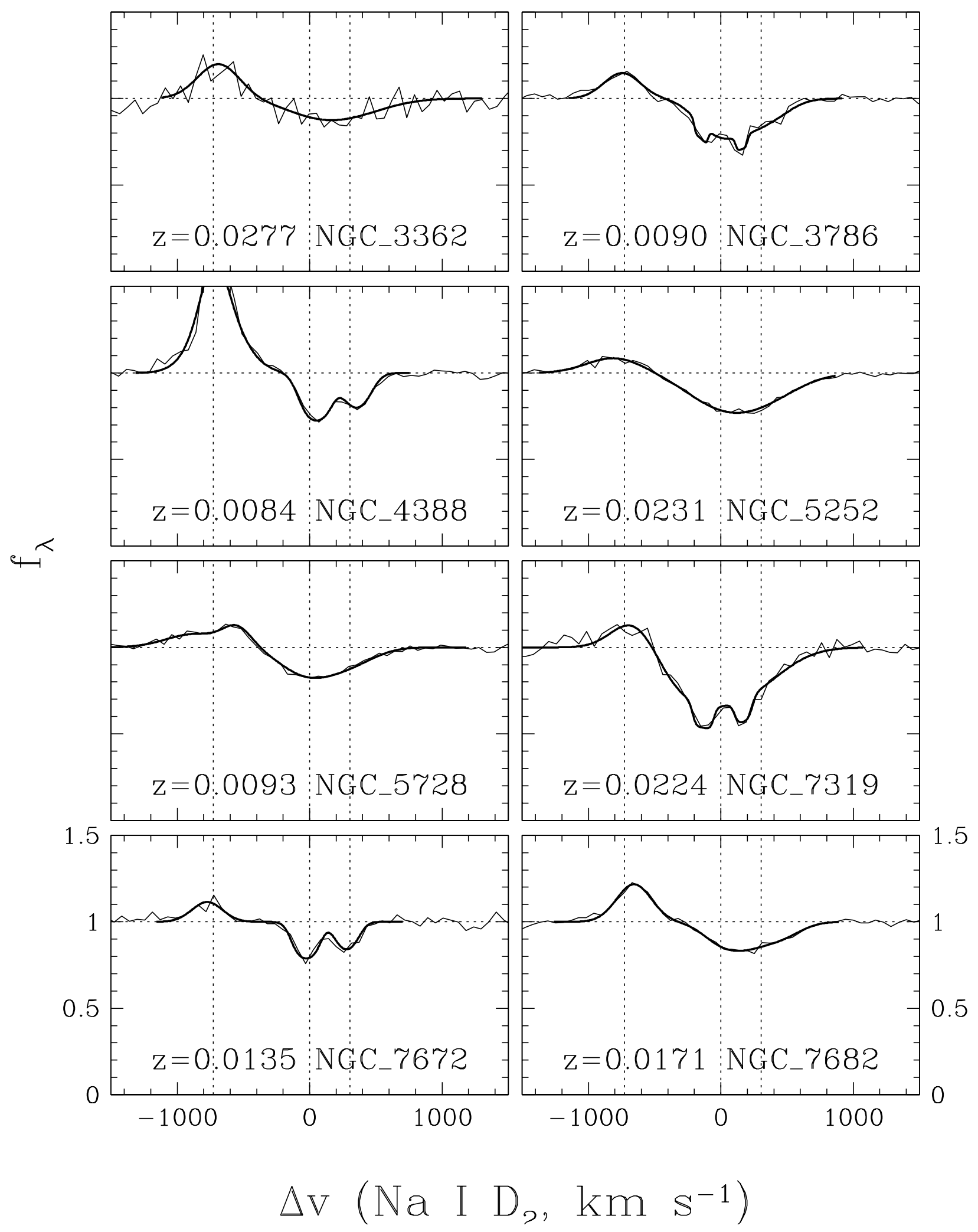

Fig. 5.-Continued 


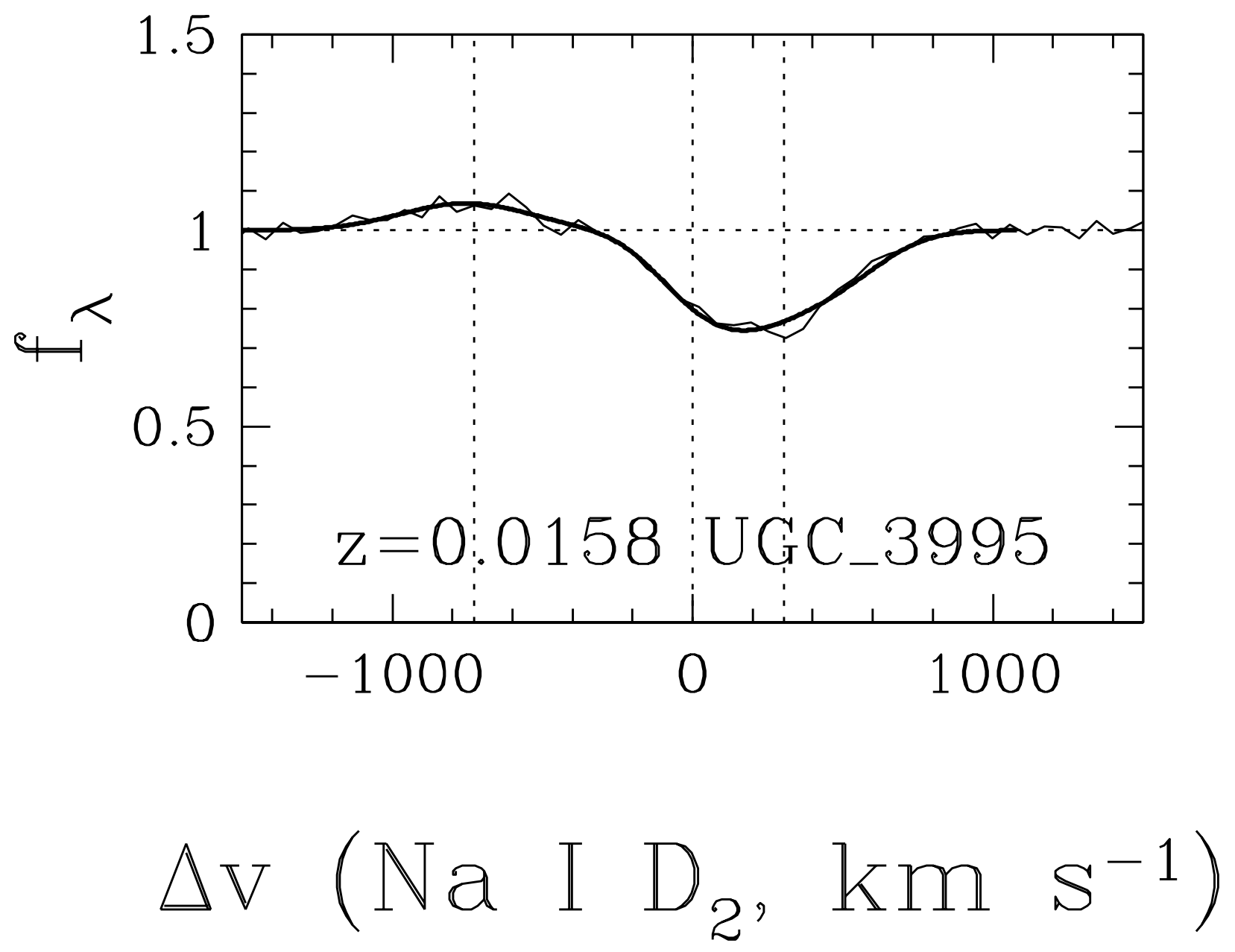

Fig. 5.-Continued 

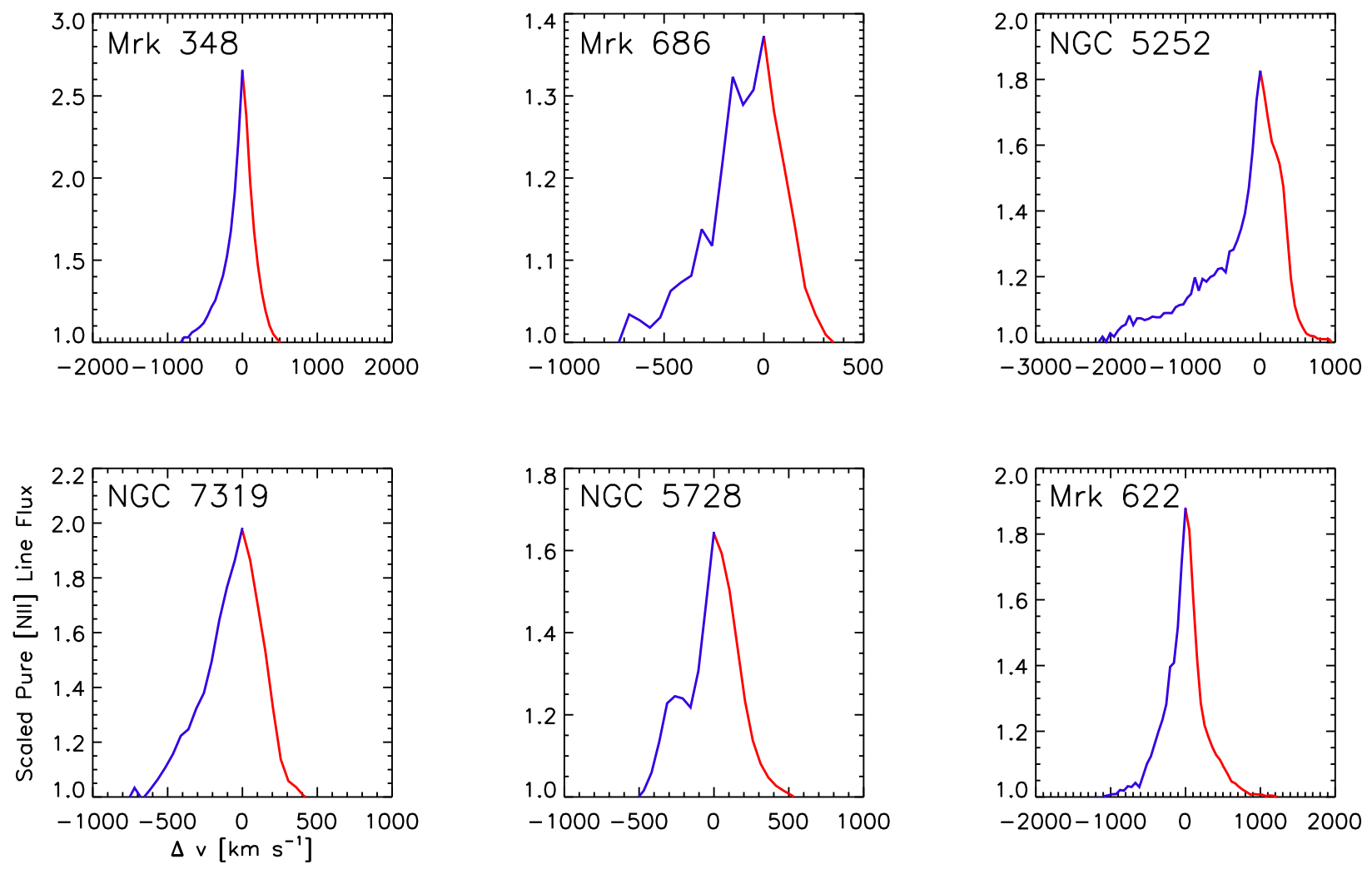

Fig. 6. - Plots showing the [N II] $\lambda 6548$ line for $\Delta v<0$ and (properly scaled) [N II] $\lambda 6583$ line for $\Delta v>0$ in 5 Seyfert 2 galaxies suspected of having blue emission-line asymmetry. The velocity scale is relative to systemic, and colors correspond to blue wing and red wing. The first four objects have noticeable blue-wing asymmetry, and the fifth (NGC 5728) shows a prominent blue bump in the emission line. The lower right panel (Mrk 622) is an example of an object which does not show an obvious asymmetry. 


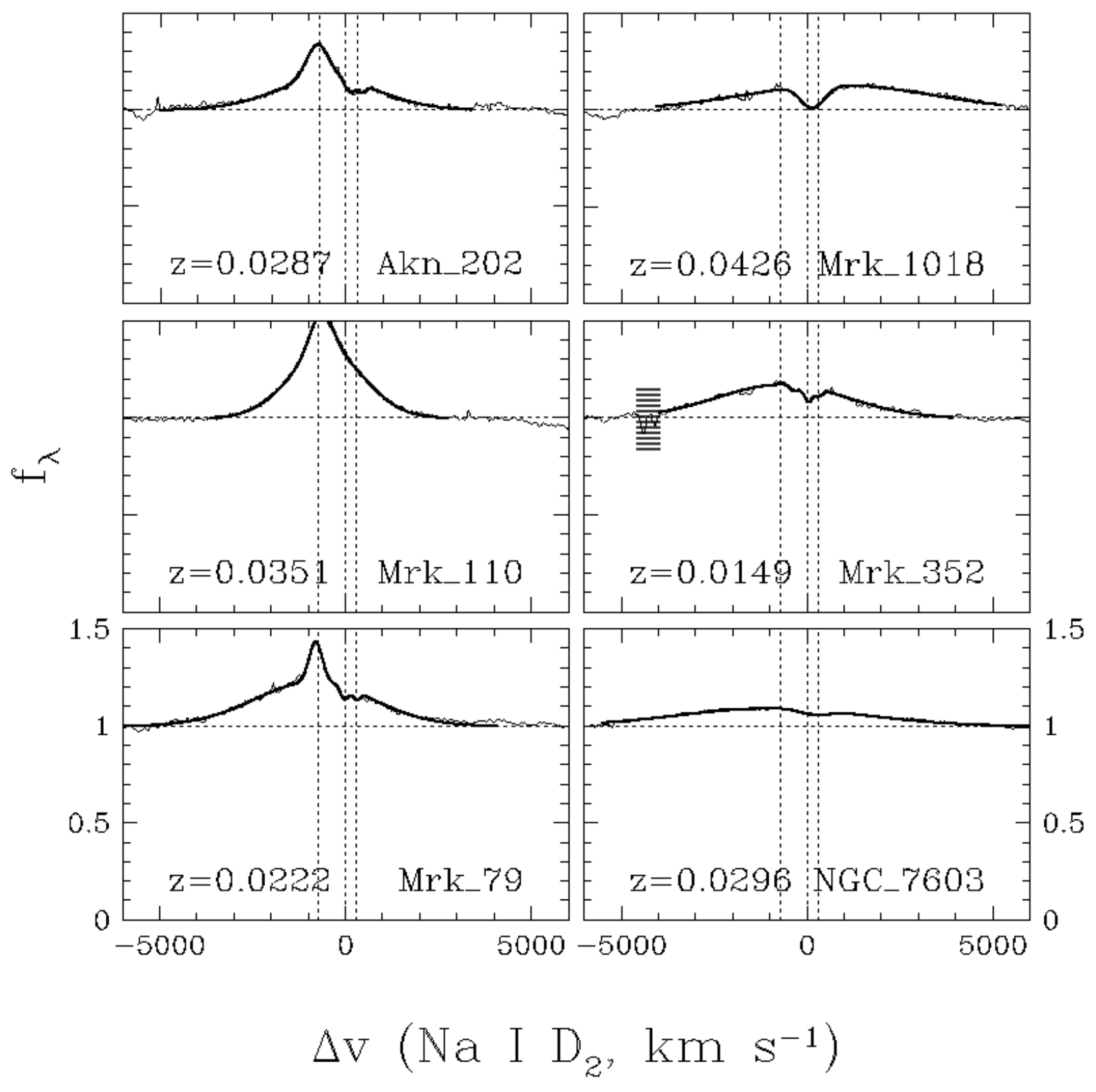

Fig. 7.- Spectra of the He I emission + Na I D absorption region in infrared-faint Seyfert 1 galaxies. See Figure 5 for details. A region of horizontal lines is used to block out atmospheric absorption in the case of Mrk 352 . 


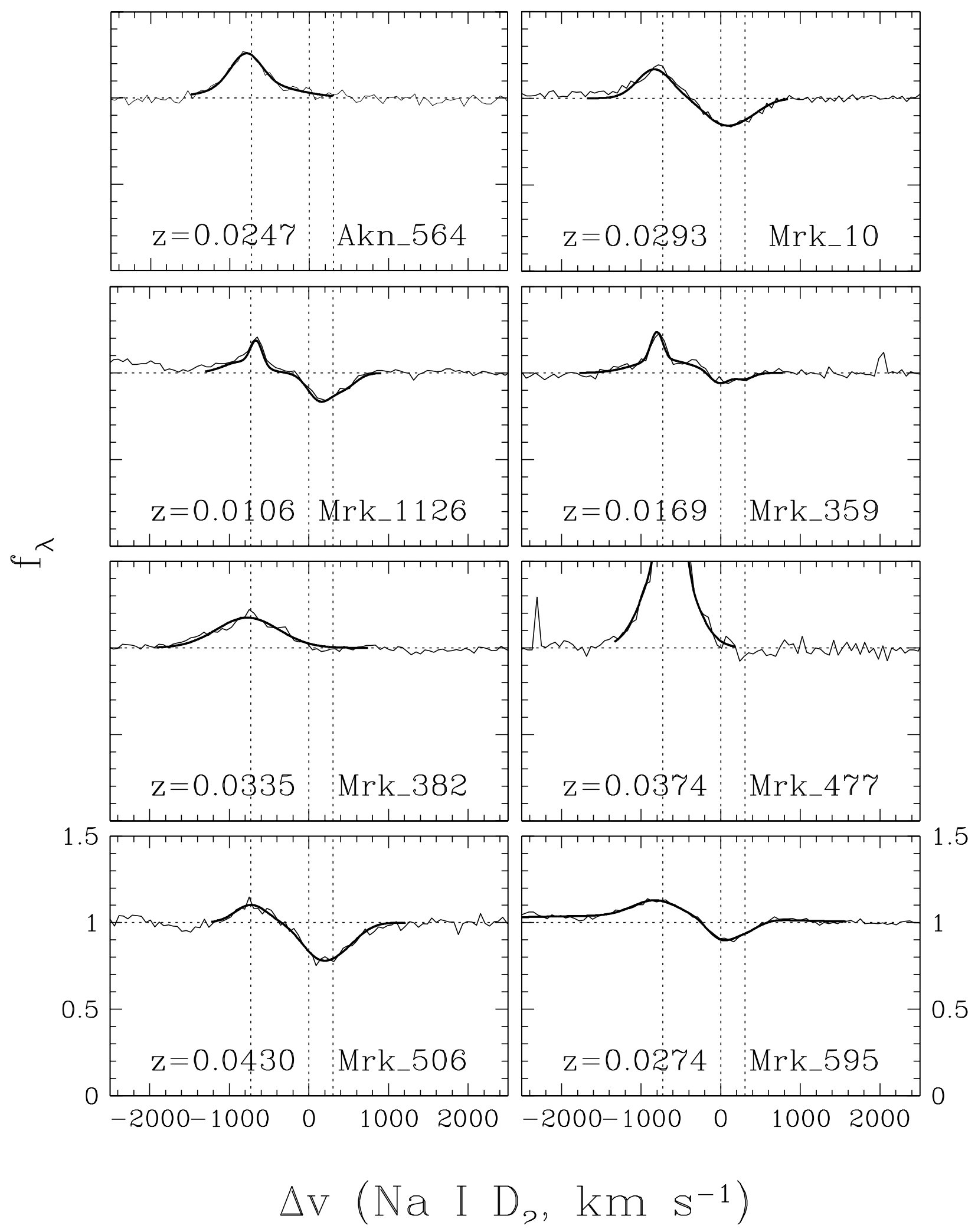

Fig. 7.-Continued 


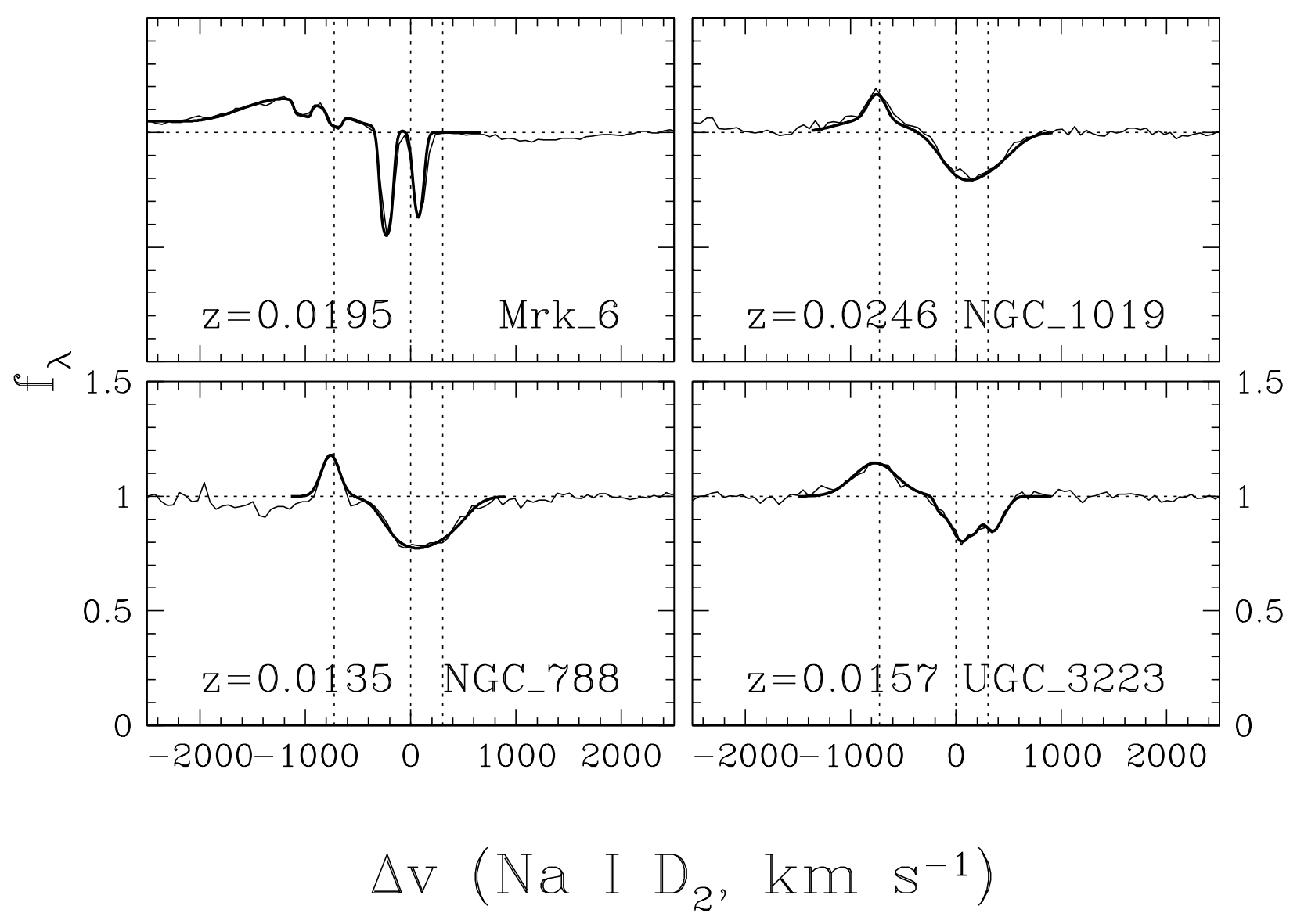

Fig. 7.-Continued 


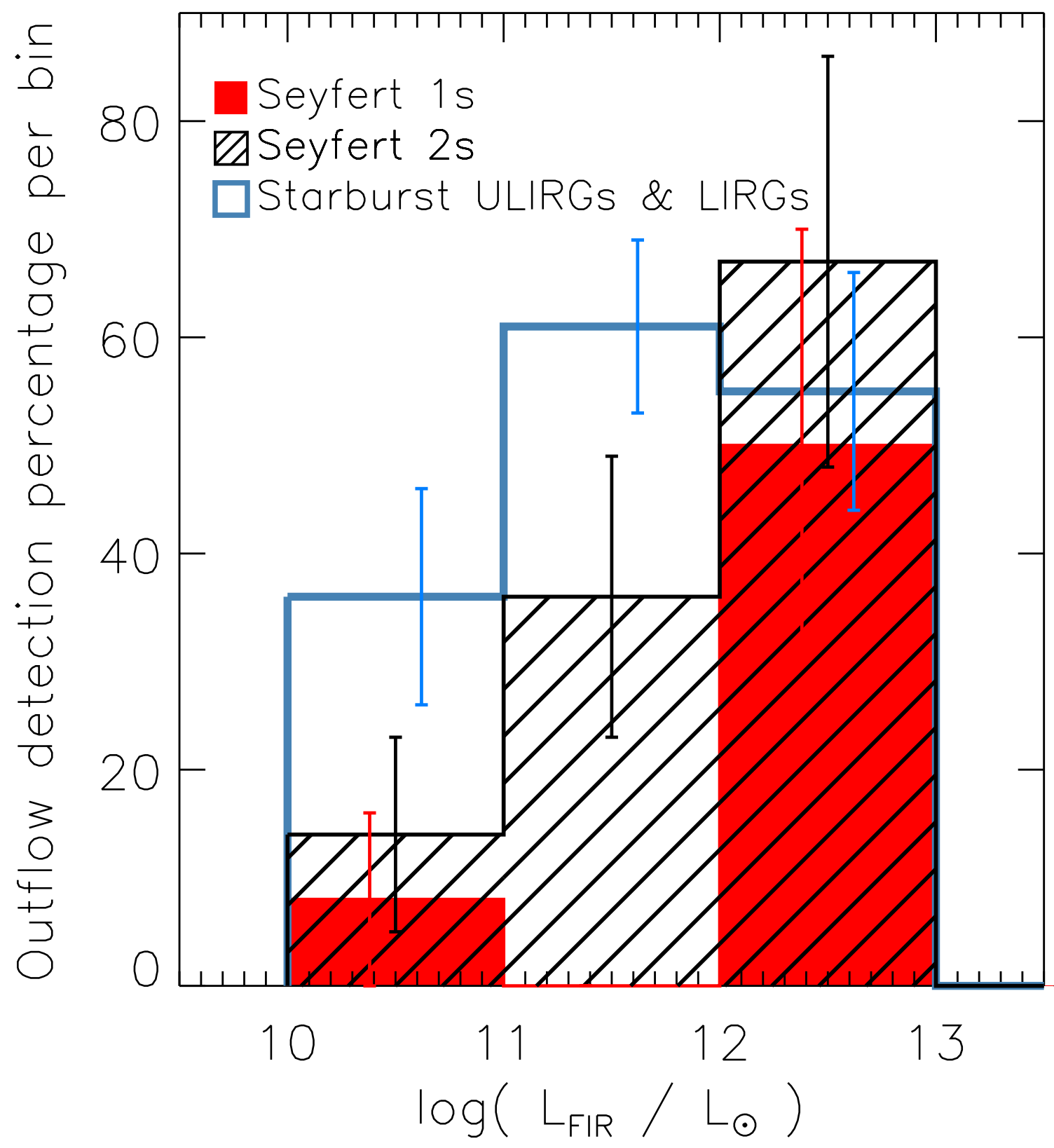

Fig. 8.- Histogram showing the fraction of Seyfert 1s, Seyfert 2s, and starburst ULIRGs and LIRGs with outflows as a function of $L_{\mathrm{FIR}}$. The Seyfert categories include both IRfaint Seyferts from this study and IR-luminous Seyferts from RVS05c. Starburst ULIRGs \& LIRGs are from RVS05b. The error bars are $1 \sigma$, assuming a binomial distribution. Note the trend of increasing outflow detection rate with increasing $L_{\mathrm{FIR}}$ for both starbursts and Seyferts. 

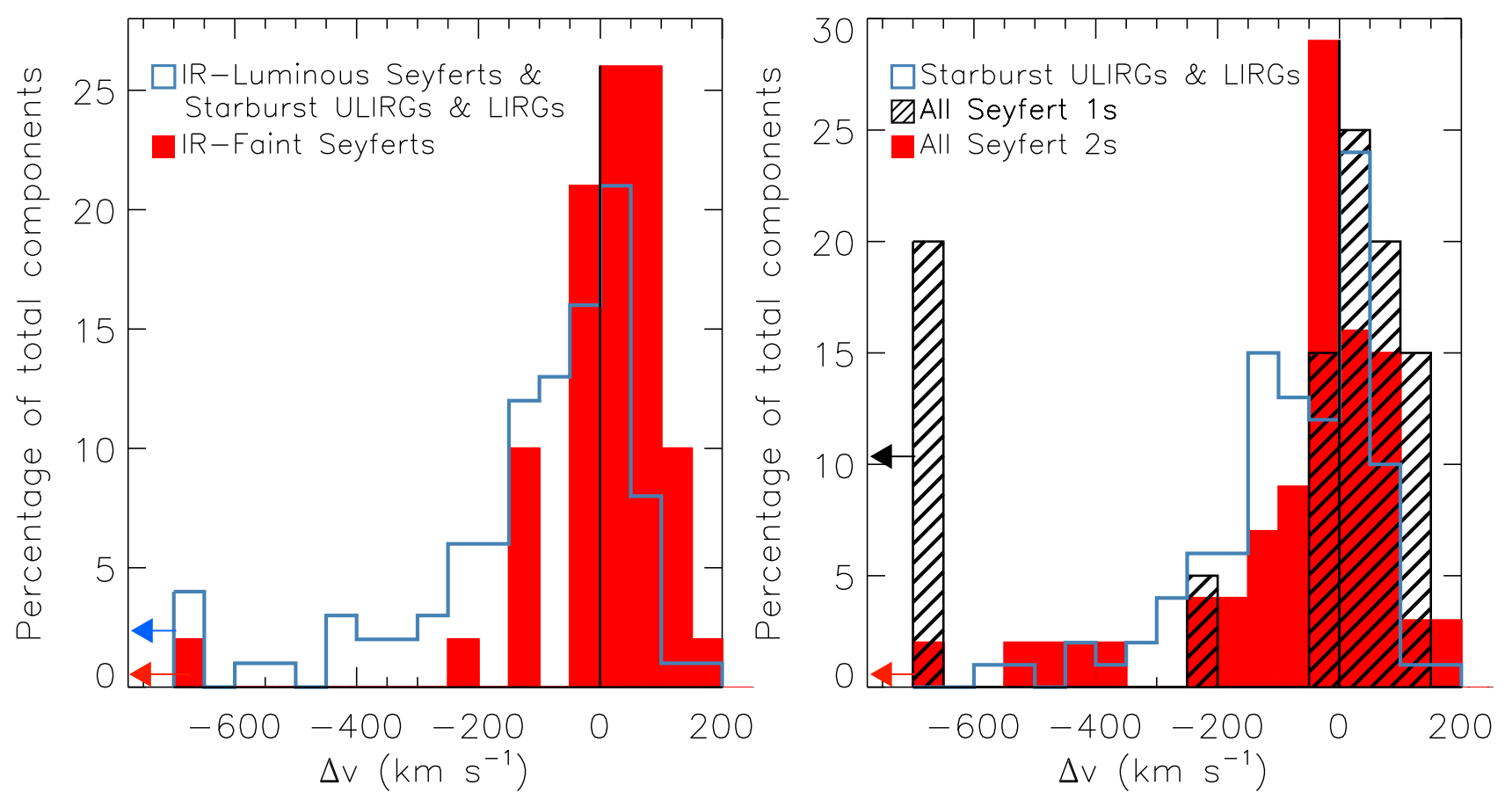

Fig. 9.- Left: Histogram showing the percentage of velocity components as a function of $\Delta v$ for the IR-faint Seyferts from this study, the IR-luminous Seyferts from RVS05c, and the starburst ULIRGs \& LIRGs from RVS05b. Objects at $\Delta v=-700 \mathrm{~km} \mathrm{~s}^{-1}$ actually have $\Delta v \leq-700 \mathrm{~km} \mathrm{~s}^{-1}$; the axis is truncated since most components have $\Delta v>-700 \mathrm{~km} \mathrm{~s}^{-1}$. Solid black line at $\Delta v=0$ highlights the division between inflows and outflows. Note the lack (excess) of outflowing (infalling) components in IR-faint Seyferts relative to the other objects. Right: Same as left, but the IR-faint Seyferts from this study and IR-luminous Seyferts from RVS05c have been combined together and divided into Seyfert types. Note the excess of infalling and high-velocity outflowing components among Seyfert 1 galaxies. 

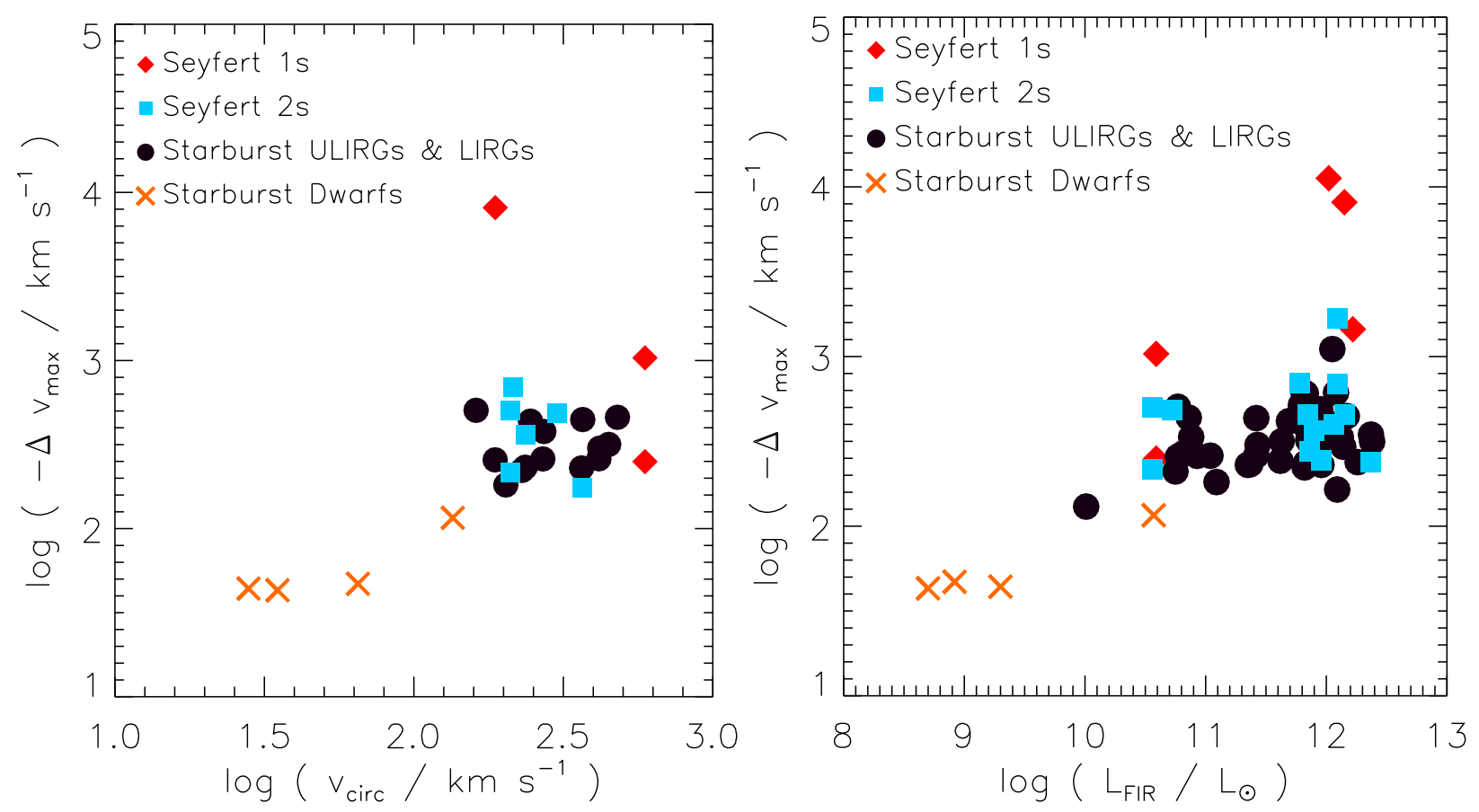

Fig. 10. - Left: Maximum outflow velocity versus circular velocity. The Seyfert 1 and Seyfert 2 categories include data from this study and from RVS05c (both IR-faint and IR-luminous Seyferts). The starburst ULIRG \& LIRG category is composed of data from RVS05b. The data on the starburst dwarfs are from Schwartz \& Martin (2004). Right: Same as left, but for maximum outflow velocity versus $L_{\mathrm{FIR}}$. The outflow kinematics in Seyfert $2 \mathrm{~s}$ (1s) are similar to (different from) those of the starburst ULIRGs and LIRGs. 

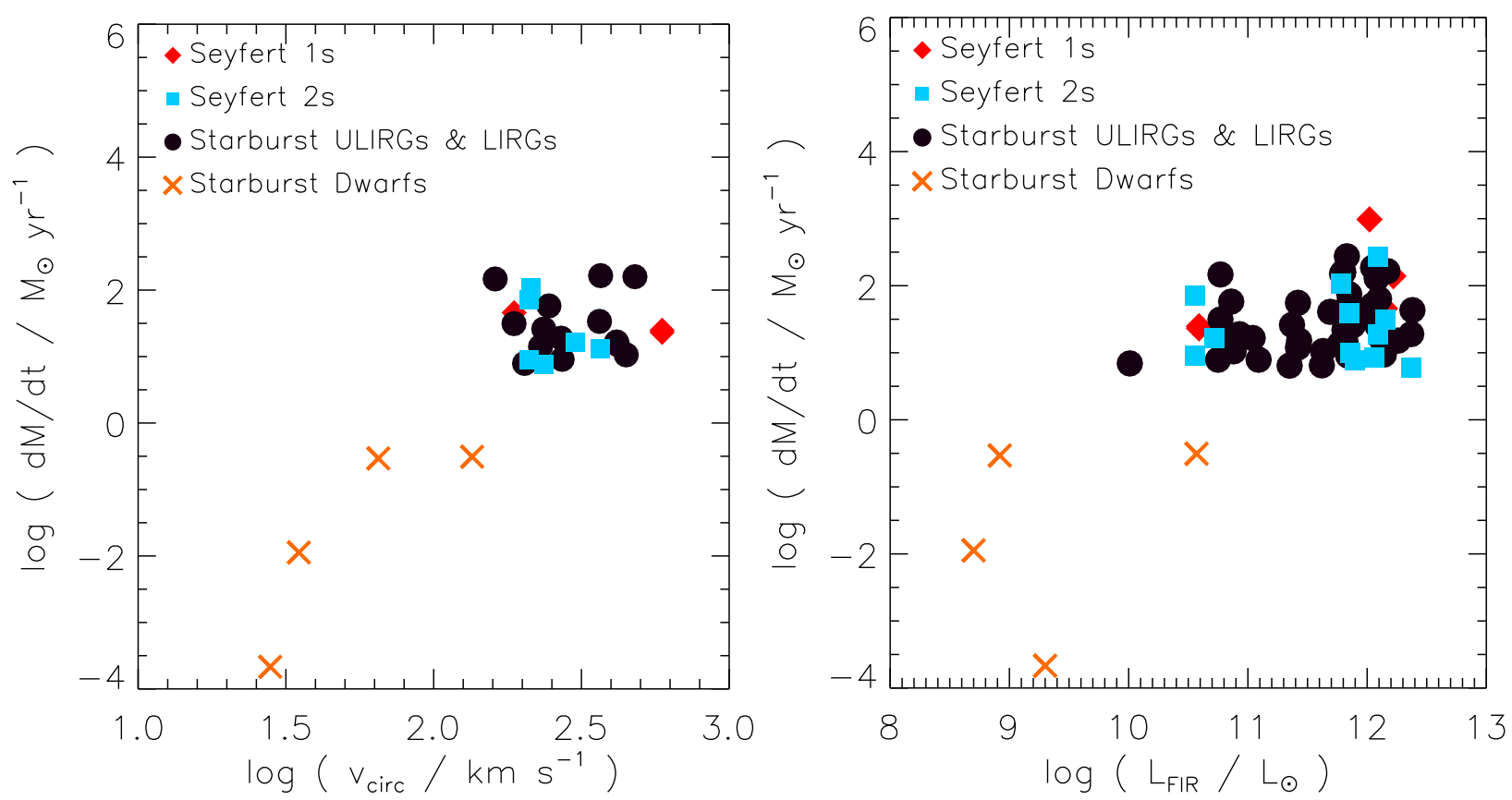

Fig. 11. - Left: Mass outflow rate versus circular velocity. See Figure 10 for descriptions of the various categories. Right: Same as left, but for mass outflow rate versus $L_{\mathrm{FIR}}$.
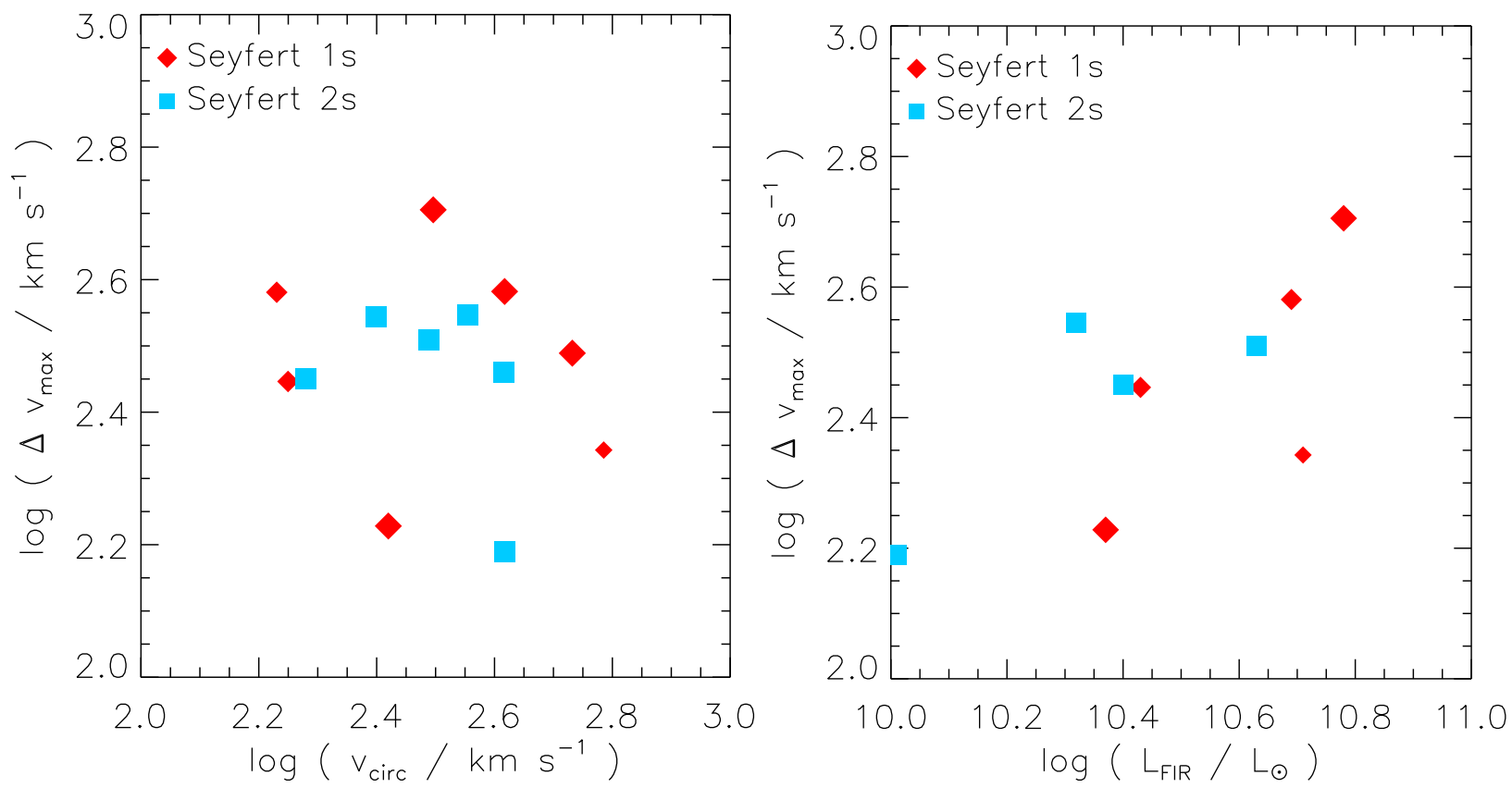

Fig. 12.- Left: $\Delta v_{\max }$ versus circular velocity for inflows detected in IR-faint Seyfert galaxies. Smaller points are those with uncertain redshifts. Right: Same as left, but for $\Delta v_{\max }$ vs. $L_{\mathrm{FIR}}$. No obvious trend is seen. 